\title{
On the Term Structure of Futures and Forward Prices.
}

\author{
Tomas Björk ${ }^{\dagger}$ \\ Department of Finance \\ Stockholm School of Economics \\ Box 6501, SE-113 83 Stockholm \\ SWEDEN \\ e-mail: tomas.bjork@hhs.se
}

\author{
Camilla Landén \\ Department of Mathematics \\ Royal Institute of Technology \\ SE-100 44 Stockholm \\ SWEDEN \\ e-mail: camilla@math.kth.se
}

December 7, 2000

\begin{abstract}
We investigate the term structure of forward and futures prices for models where the price processes are allowed to be driven by a general marked point process as well as by a multidimensional Wiener process. Within an infinite dimensional HJM-type model for futures and forwards we study the properties of futures and forward convenience yield rates. For finite dimensional factor models, we develop a theory of affine term structures, which is shown to include almost all previously known models. We also derive two general pricing formulas for futures options. Finally we present an easily applicable sufficient condition for the possibility of fitting a finite dimensional futures price model to an arbitrary initial futures price curve, by introducing a time dependent function in the drift term.
\end{abstract}

Key words: term structure, futures price, forward price, options, jump-diffusion model, affine term structure.

JEL classification: E43, G13

\footnotetext{
*We are grateful to K. Miltersen for some very helpful comments.

${ }^{\dagger}$ The financial support of ITM is gratefully acknowledged.
} 


\section{Contents}

1 Introduction $\quad 2$

2 Basics 3

3 Modeling the forward and futures convenience yield $\quad 7$

3.1 Basic definitions . . . . . . . . . . . . . . . . . . 7

3.2 Elementary properties ................. . . 8

3.3 Drift conditions for the yields . . . . . . . . . . . . . . 11

4 Affine term structures for forwards and futures 14

4.1 Futures .................... . . 14

4.2 Examples ... . . . . . . . . . . . . . . . 17

4.2.1 The Schwartz spot price model. . . . . . . . . . . . 17

4.2.2 The general affine Wiener driven spot price model. . . . . 17

4.2.3 The Gibson-Schwartz two-factor model. . . . . . . . . . . 18

4.2.4 The Schwartz three-factor model. . . . . . . . . . . . . . . 19

4.2.5 The Hilliard-Reis three-factor model. . . . . . . . . . . . 20

4.2.6 A three-factor model with positive short rate . . . . . . 21

4.3 Forwards ..................... . . 22

5 Options on futures prices $\quad 24$

5.1 General formula . . . . . . . . . . . . . . . . . . . . . . 24

5.2 Special cases . . . . . . . . . . . . . . . . 28

5.2.1 Deterministic volatilities . . . . . . . . . . . . 28

5.2.2 The Hilliard-Reis three-factor model. . . . . . . . . . . . 29

5.2.3 The Gaussian case . . . . . . . . . . . . . . . . 31

5.2.4 Quadratic interest rates .............. 32

6 Inverting the term structure $\quad 35$

6.1 Conditionally affine models . . . . . . . . . . . . . . 35

6.2 An example ...................... 39

\section{Introduction}

The object of this paper is to study the properties of forward and futures prices (as well as their derivatives) within a reasonably general framework, and in particular we are interested in the case when the underlying asset is non-financial, i.e. when we have a non zero convenience yield.

The literature on forward and futures contracts is a rich one. In [1], [5], [10], [17], and [18] the models have purely Wiener driven price dynamics, while [11] also allows for a point process. In this paper we are in particular inspired by the exposition in [17].

The main contributions of the present paper are as follows. 
- In Section 2 we present a general framework for the term structure dynamics of forward and futures prices, allowing for a general marked point process as well as for a multidimensional Wiener process in the price dynamics. The approach is to model the entire term structure a la HeathJarrow-Morton, and the main novelty is the introduction of the point process.

- In Section 3 we study how it is possible to model the forward and futures term structure by modeling the term structure of forward and futures convenience yield rates. This approach has earlier been taken in [1] and [17]. We extend the earlier results to the point process case, and we also give new (even for the pure Wiener case) results about the forward convenience yield rates drift condition. Furthermore we provide new results on the relations between forward and futures convenience yield rates and the conditional expectation of the future value of the spot convenience yield.

- In Section 4 we consider finite dimensional factor models and develop a theory of affine term structures for forward and futures prices. This is done very much as in the interest rate case (see [7]) and we show that almost all previously known factor models for forwards and futures belong to the affine class. In particular we show that the natural (from an affine point of view) spot price models are the ones where the local rate of return and the squared volatility are affine in the log of the spot price. We also provide new affine factor models.

- Section 5 is devoted to the pricing of futures options within the general framework of Section 2. Since the futures price process is not the spot price process of a traded asset, the general Geman-El Karoui-Rochet option pricing formula (see [9]) is not applicable. Instead, by introducing two hitherto new types of martingale measures, we mange to provide two different general option pricing formulas and we also discuss the economic interpretation of these formulas.

- Finally, in Section 6 we discuss the problem of fitting a given finite dimensional factor model to a given initial futures term structure. We present a reasonably large class of models for which the fitting can be done by means of a deterministic perturbation of the drift term of the spot price, and it is seen that most existing models in the literature belong to this class.

\section{Basics}

We consider a financial market living on a stochastic basis (filtered probability space) $(\Omega, \mathcal{F}, \mathbf{F}, Q)$ where $\mathbf{F}=\left\{\mathcal{F}_{t}\right\}_{t \geq 0}$. The basis is assumed to carry a multidimensional Wiener process $W$ as well as a marked point process $\mu(d t, d x)$ on a measurable Lusin mark space $(E, \mathcal{E})$ with predictable compensator $\nu(d t, d x)$. The predictable $\sigma$-algebra is denoted by $\mathcal{Q}$, and we make the definition $\tilde{\mathcal{Q}}=$ 
$\mathcal{Q} \otimes \mathcal{E}$. We assume that $\nu([0, t] \times E)<\infty Q$-a.s. for all finite $t$, i.e. $\mu$ is a multivariate point process in the terminology of [13]. For simplicity we also assume that $\mu$ has an intensity $\lambda$, i.e. the compensator has the form

$$
\nu(d t, d y)=\lambda(t, d y) d t .
$$

The compensated point process $\tilde{\mu}$ is defined by $\mu(d t, d x)=\mu(d t, d y)-\nu(d t, d y)$.

The primitive assets to be considered on the market are forward and futures contracts, written on a given underlying asset, with different delivery dates. We denote the forward price at time $t$ of a forward contract with delivery date $T$ by $G(t, T)$. The futures price at time $t$ with delivery date $T$ is denoted by $F(t, T)$. The induced spot price process $S_{t}$ is given by a standard arbitrage argument as

$$
S_{t}=F(t, t)=G(t, t)
$$

We assume that there is an idealized market (liquid, frictionless, unlimited short selling allowed etc.) for forward and futures contracts for every delivery date $T$. We do, however, not assume that the asset underlying the futures and forward market is traded on an idealized market. The market for the underlying could for example be very thin, there could be transactions costs, prohibitive storage costs or shortselling constraints. Typical examples would be a commodity market or a market for electric energy. We will also have to consider the bond market, and we let $p(t, T)$ denote the price, at time $t$, of a zero coupon bond maturing at $T$. The corresponding forward rates are denoted by $f(t, T)$, where as usual

$$
f(t, T)=-\frac{\partial}{\partial T} \ln p(t, T) .
$$

The short rate is denoted by $r(t)$, and defined by $r(t)=f(t, t)$. The money account is defined as usual by $B(t)=\exp \int_{0}^{t} r(s) d s$. We assume that the market for bonds, futures and forwards is arbitrage free in the sense that the probability measure $Q$ is a martingale measure (for the numeraire $B$ ) for the economy. For the rest of the paper we will, either by implication or by assumption, consider dynamics of the following type.

\section{Forward price dynamics}

$$
\begin{aligned}
d G(t, T) & =G(t, T) \alpha_{G}(t, T) d t+G(t, T) \sigma_{G}(t, T) d W_{t} \\
& +G(t-, T) \int_{E} \delta_{G}(t, y, T) \mu(d t, d y),
\end{aligned}
$$

Futures price dynamics

$$
\begin{aligned}
d F(t, T) & =F(t, T) \alpha_{F}(t, T) d t+F(t, T) \sigma_{F}(t, T) d W_{t} \\
& +F(t-, T) \int_{E} \delta_{F}(t, y, T) \mu(d t, d y)
\end{aligned}
$$


Spot price dynamics

$$
\begin{aligned}
d S(t) & =S(t) \alpha_{S}(t) d t+S(t) \sigma_{S}(t) d W_{t} \\
& +S(t-) \int_{E} \delta_{S}(t, y) \mu(d t, d y)
\end{aligned}
$$

Short rate dynamics

$$
d r(t)=\alpha_{r}(t) d t+\sigma_{r}(t) d W_{t}+\int_{E} \delta_{r}(t, y) \mu(d t, d y)
$$

Bond price dynamics

$$
\begin{aligned}
d p(t, T) & =p(t, T) \alpha_{p}(t, T) d t+p(t, T) \sigma_{p}(t, T) d W_{t} \\
& +p(t-, T) \int_{E} \delta_{p}(t, y, T) \mu(d t, d y)
\end{aligned}
$$

Forward rate dynamics

$$
d f(t, T)=\alpha_{f}(t, T) d t+\sigma_{f}(t, T) d W_{t}+\int_{E} \delta_{f}(t, y, T) \mu(d t, d y) .
$$

In the above formulas the coefficient processes are assumed to meet standard conditions required to guarantee that the various processes are well defined.

We recall the following basic results (see e.g. [2]).

Proposition 2.1 Let $Q^{T}$ denote the T-forward martingale measure. Then the following hold.

- For a fixed $T$, the futures price process $F(t, T)$ is a $Q$-martingale, and in particular we have

$$
F(t, T)=E^{Q}\left[S(T) \mid \mathcal{F}_{t}\right]
$$

- The forward price process $G(t, T)$ is a martingale under $Q^{T}$, and in particular we have

$$
G(t, T)=E^{Q^{T}}\left[S(T) \mid \mathcal{F}_{t}\right] .
$$

For future use, we recall the following relation between the volatilities of the forward rates and the bond prices.

Proposition 2.2 With notation as in (7)-(8) we have

$$
\begin{aligned}
\sigma_{p}(t, T) & =-\int_{t}^{T} \sigma_{f}(t, s) d s, \\
\delta_{p}(t, y, T) & =e^{-D_{f}(t, y, s)}-1,
\end{aligned}
$$

where

$$
D_{f}(t, T, y)=\int_{t}^{T} \delta_{f}(t, y, s) d s
$$




\section{Proof. See [4].}

Since the modeling above is done directly under a martingale measure, there will be "drift conditions", relating the drift terms to the volatilities of the various processes above.

Proposition 2.3 Under the martingale measure $Q$, the following relations hold.

$$
\begin{aligned}
\alpha_{G}(t, T) & =\sigma_{G}(t, T) \int_{t}^{T} \sigma_{f}^{\star}(t, s) d s-\int_{E} \delta_{G}(t, y, T) e^{-D_{f}(t, y, T)} \lambda(t, d y),(14) \\
\alpha_{F}(t, T) & =-\int_{E} \delta_{F}(t, y, T) \lambda(t, d y) \\
\alpha_{p}(t, T) & =r_{t}-\int_{E}\left\{e^{-D_{f}(t, y, T)}-1\right\} \lambda(t, d y) \\
\alpha_{f}(t, T) & =\sigma_{f}(t, T) \int_{t}^{T} \sigma_{f}^{\star}(t, s) d s-\int_{E} \delta_{f}(t, y, T) e^{-D_{f}(t, y, T)} \lambda(t, d y)
\end{aligned}
$$

Proof. For (16)-(17) we refer to [4]. In order to derive (15), we rewrite the $F$-dynamics on semimartingale form by compensating the point process. We thus obtain

$$
\begin{aligned}
d F(t, T) & =F(t, T)\left\{\alpha_{F}(t, T)+\int_{E} \delta_{F}(t, y, T) \lambda(t, d y)\right\} d t \\
& +F(t, T) \sigma_{F}(t, T) d W_{t} \\
& +F(t-, T) \int_{E} \delta_{F}(t, y, T) \tilde{\mu}(d t, d y)
\end{aligned}
$$

This formula gives the futures price process as a sum of a predictable finite variation process and two martingales. Since the futures price process is a $Q$ martingale, the $d t$-term must vanish, and we are finished.

To derive the drift condition for the forward price process, we now change measure from $Q$ to the $T$-forward measure $Q^{T}$. From general theory (see [9]) we know that the likelihood process for this measure transformation is given by

$$
L_{t}^{T}=\frac{p(t, T)}{B(t) p(0, T)}
$$

where

$$
L_{t}^{T}=\frac{d Q^{T}}{d Q}, \text { on } \mathcal{F}_{t}
$$

Using (12), (16) and the Itô formula, we have the $L^{T}$-dynamics

$$
d L_{t}^{T}=L_{t}^{T} \sigma_{p}(t, T) d W_{t}+L_{t-}^{T} \int_{E} \delta_{p}(t, y, T) \mu(d t, d y) .
$$


From the Girsanov Theorem (see [13]) it now follows that we can write

$$
d W_{t}=\sigma_{p}^{\star}(t, T) d t+d W_{t}^{T},
$$

where $W^{T}$ is a $Q^{T}$-Wiener process. It also follows from the Girsanov Theorem that, under $Q^{T}$, the point process $\mu$ has an intensity $\lambda^{T}$ given by

$$
\lambda^{T}(t, d y)=\left(\delta_{p}(t, y, T)+1\right) \lambda(t, d y) .
$$

We can thus write the $Q^{T}$-semimartingale dynamics for $G(t, T)$ as

$$
\begin{aligned}
d G(t, T) & =G(t, T)\left\{\alpha_{G}(t, T)+\sigma_{G}(t, T) \sigma_{p}^{\star}(t, T)\right. \\
& \left.+\int_{E} \delta_{G}(t, y, T)\left(\delta_{p}(t, y, T)+1\right) \lambda(t, d y)\right\} d t \\
& +G(t, T) \sigma_{G}(t, T) d W_{t}^{T} \\
& +G(t-, T) \int_{E} \delta_{G}(t, y, T)\left\{\mu(d t, d y)-\lambda^{T}(t, d y) d t\right\} .
\end{aligned}
$$

Since $G(t, T)$ is a $Q^{T}$-martingale the $d t$-term thus has to vanish and, using Proposition 2.2, we obtain (14).

\section{$3 \quad$ Modeling the forward and futures convenience yield}

In this section we will study how it is possible to model the term structure of forwards and futures by modeling the spot price and the term structure of the forward and futures convenience yields. This approach goes back to [1], [5] and [17].

\subsection{Basic definitions}

Definition 3.1 With notations as above we define the following objects.

- The term structure of futures convenience yields $\varphi(t, T)$, for $0 \leq t \leq T$, is defined by the relation

$$
F(t, T)=S(t) e^{\int_{t}^{T}[f(t, s)-\varphi(t, s)] d s} .
$$

- The term structure of forward convenience yields $\gamma(t, T)$, for $0 \leq t \leq T$, is defined by the relation

$$
G(t, T)=S(t) e^{\int_{t}^{T}[f(t, s)-\gamma(t, s)] d s} .
$$


- The spot convenience yield $c(t)$ is defined by the relation

$$
\alpha_{S}(t)=r(t)-c(t)-\int_{E} \delta_{S}(t, x) \lambda(t, d x)
$$

In order to connect with elementary theory we note that, for the case when $S(t)$ is the price of an underlying asset traded on an idealized market, we have the relation

$$
G(t, T)=S(t) p(t, T)^{-1}=G(t, T)=S(t) e^{\int_{t}^{T} f(t, s) d s} .
$$

The forward convenience yields thus measures the deviation from this idealized situation. We also note that, by the definition above, the spot price has the $Q$-dynamics

$$
d S_{t}=S(t)\left(r_{t}-c_{t}\right) d t+S(t) \sigma_{S}(t) d W+\int_{E} \delta_{S}(t, x, T) \tilde{\mu}(d t, d x) .
$$

Thus, as usual, under the martingale measure, the local mean rate of return of the spot price equals the short rate minus the spot convenience yield.

We will start by investigating some elementary properties of the various yields, and then we go on to discuss how to model the yield dynamics.

\subsection{Elementary properties}

It is easy to see that if the spot convenience yield $c_{t}$ is deterministic, then we have, for all $0 \leq t \leq T$,

$$
c_{T}=\gamma(t, T) .
$$

In the general situation with stochastic $c_{t}$, this result raises the question if, $\gamma(t, T)$ and/or $\varphi(t, T)$ can be viewed as predictors (at time $t$ ) of the spot yield at time $T$. The following result provides an answer to this question. In order to shorten notation, we use $\operatorname{Cov}_{t}^{Q}$ to denote the $Q$-covariance, conditioned on $\mathcal{F}_{t}$.

\section{Proposition 3.1}

- The forward convenience yield satisfies

$$
\gamma(t, T)=E^{Q}\left[c_{T} \mid \mathcal{F}_{t}\right]+\frac{\operatorname{Cov}_{t}^{Q}\left[e^{-\int_{t}^{T} r(s) d s} S_{T}, c_{T}\right]}{E^{Q}\left[e^{-\int_{t}^{T} r(s) d s} S_{T} \mid \mathcal{F}_{t}\right]}
$$

- The futures convenience yield satisfies

$$
\begin{aligned}
\varphi(t, T) & =E^{Q}\left[c_{T} \mid \mathcal{F}_{t}\right]+\frac{\operatorname{Cov}_{t}^{Q}\left[S_{T}, c_{T}\right]}{E^{Q}\left[S_{T} \mid \mathcal{F}_{t}\right]} \\
& +f(t, T)-E^{Q}\left[r_{T} \mid \mathcal{F}_{t}\right]-\frac{\operatorname{Cov}_{t}^{Q}\left[S_{T}, r_{T}\right]}{E^{Q}\left[S_{T} \mid \mathcal{F}_{t}\right]}
\end{aligned}
$$


- In particular we have

$$
c_{t}=\varphi(t, t)=\gamma(t, t) .
$$

Proof. We start by noting that (26) follows immediately from (24) and (25). From the definition of $\gamma$ we have

$$
-\frac{\partial}{\partial T} \ln G(t, T)=\gamma(t, T)-f(t, T) .
$$

From general theory we also know that

$$
\begin{aligned}
G(t, T) & =\frac{1}{p(t, T)} E^{Q}\left[e^{-\int_{t}^{T} r(s) d s} S(T) \mid \mathcal{F}_{t}\right] \\
& =e^{\int_{t}^{T} f(t, s) d s} E^{Q}\left[e^{-\int_{t}^{T} r(s) d s} S(T) \mid \mathcal{F}_{t}\right]
\end{aligned}
$$

Using subscript to denote partial derivatives, we obtain

$$
G_{T}(t, T)=f(t, T) G(t, T)-e^{\int_{t}^{T} f(t, s) d s} \frac{\partial}{\partial T} E^{Q}\left[e^{-\int_{t}^{T} r(s) d s} S(T) \mid \mathcal{F}_{t}\right]
$$

In order to compute the last term, let us (for a fixed $t$ ) define the process $Z_{T}$ by $Z_{T}=e^{-\int_{t}^{T} r(s) d s} S(T)$. An application of the Itô formula, together with (23), gives us

$$
\begin{aligned}
d Z_{T} & =-r_{T} Z_{T} d T+e^{-\int_{t}^{T} r(s) d s} d S_{T} \\
& =-r_{T} Z_{T} d T+e^{-\int_{t}^{T} r(s) d s} S_{T}\left(r_{T}-c_{T}\right) d T+d M \\
& =-e^{-\int_{t}^{T} r(s) d s} S_{T} c_{T} d T+d M,
\end{aligned}
$$

where $d M$ denotes a generic $Q$-martingale increment. From this we obtain

$$
\begin{aligned}
\frac{\partial}{\partial T} E^{Q}\left[e^{-\int_{t}^{T} r(s) d s} S(T) \mid \mathcal{F}_{t}\right] & =\lim _{h \rightarrow 0} \frac{1}{h} E^{Q}\left[Z_{T+h}-Z_{T} \mid \mathcal{F}_{t}\right] \\
& =-\lim _{h \rightarrow 0} \frac{1}{h} E^{Q}\left[\int_{T}^{T+h} e^{-\int_{t}^{u} r(s) d s} S_{u} c_{u} d u \mid \mathcal{F}_{t}\right] \\
& =-E^{Q}\left[e^{-\int_{t}^{T} r(s) d s} S_{T} c_{T} \mid \mathcal{F}_{t}\right] .
\end{aligned}
$$

We thus obtain

$$
-\frac{\partial}{\partial T} \ln G(t, T)=\frac{G_{T}(t, T)}{G(t, T)}=-f(t, T)+\frac{E^{Q}\left[e^{\int_{t}^{T} r(s) d s} c_{T} S_{T} \mid \mathcal{F}_{t}\right]}{E^{Q}\left[e^{\int_{t}^{T} r(s) d s} S_{T} \mid \mathcal{F}_{t}\right]} .
$$


Comparing this expression with (27) gives us

$$
\gamma(t, T)=\frac{E^{Q}\left[e^{\int_{t}^{T} r(s) d s} c_{T} S_{T} \mid \mathcal{F}_{t}\right]}{E^{Q}\left[e^{\int_{t}^{T} r(s) d s} S_{T} \mid \mathcal{F}_{t}\right]} .
$$

Using the formula $E[X Y]=E[X] \cdot E[Y]+\operatorname{Cov}(X, Y)$, this proves (24).

For the futures convenience yield we have, from (20),

$$
-\frac{\partial}{\partial T} \ln F(t, T)=\varphi(t, T)-f(t, T) .
$$

From general theory we also have

$$
F(t, T)=E^{Q}\left[S_{T} \mid \mathcal{F}_{t}\right] .
$$

From this we obtain (arguing as above)

$$
F_{T}(t, T)=E^{Q}\left[S_{T}\left(c_{T}-r_{T}\right) \mid \mathcal{F}_{t}\right] .
$$

Thus we have

$$
-\frac{\partial}{\partial T} \ln F(t, T)=\frac{E^{Q}\left[S_{T}\left(c_{T}-r_{T}\right) \mid \mathcal{F}_{t}\right]}{E^{Q}\left[S_{T} \mid \mathcal{F}_{t}\right]}
$$

so

$$
\varphi(t, T)-f(t, T)=\frac{E^{Q}\left[S_{T}\left(c_{T}-r_{T}\right) \mid \mathcal{F}_{t}\right]}{E^{Q}\left[S_{T} \mid \mathcal{F}_{t}\right]}
$$

which proves (25).

We also have some easy consequences from this result.

\section{Corollary 3.1}

- If the spot convenience yield $c$ is deterministic, then

$$
\gamma(t, T)=c_{T}, \quad \forall 0 \leq t \leq T .
$$

- If the short rate $r$ is deterministic, then

$$
\varphi(t, T)=\gamma(t, T)=E^{Q}\left[c_{T} \mid \mathcal{F}_{t}\right]+\frac{\operatorname{Cov}_{t}^{Q}\left[S_{T}, c_{T}\right]}{E^{Q}\left[S_{T} \mid \mathcal{F}_{t}\right]} .
$$

Proof. The relation (28) follows immediately from (24). The formula (29) follows also from (28) together with the fact that $F=G$ (and thus $\varphi=\gamma$ ) when interest rates are deterministic. 


\subsection{Drift conditions for the yields}

By specifying the dynamics of the spot price and the futures (forward) convenience yield, the dynamics of futures (forward) prices are completely specified. Since we are modeling under a martingale measure we will have the drift conditions for futures and forward price dynamics given by Proposition 2.3, and these conditions will obviously imply drift conditions on the yield dynamics. Before starting on this investigation we need a small technical lemma.

Lemma 3.1 Assume that, for each $T$ the process $X(t, T)$ has dynamics, for $t \leq T$, given by

$$
\begin{aligned}
d X(t, T) & =\alpha_{X}(t, T) d t+\sigma_{X}(t, T) d W_{t} \\
& +\int_{E} \delta_{X}(t, y, T) \mu(d t, d y)
\end{aligned}
$$

where the coefficient processes are assumed to meet standard conditions required to guarantee that the $X$ process is well defined. Assume furthermore that the coefficients are regular enough to allow for an application of the stochastic Fubini Theorem. If, for every $T$, the process $Z(t, T)$ is defined by

$$
Z(t, T)=\int_{t}^{T} X(t, s) d s
$$

then the stochastic differential of $Z$ is given by

$$
d Z(t, T)=\left\{A_{X}(t, T)-X(t, t)\right\} d t+S_{X}(t, T) d W_{t}+\int_{E} D(t, y, T) \mu(d t, d y),
$$

where

$$
\begin{aligned}
& A_{X}(t, T)=\int_{t}^{T} \alpha_{X}(t, s) d s \\
& S_{X}(t, T)=\int_{t}^{T} \sigma_{X}(t, s) d s \\
& D_{X}(t, T)=\int_{t}^{T} \delta_{X}(t, s) d s .
\end{aligned}
$$

\section{Proof. Fubini.}

We may now state and prove the martingale measure drift conditions for the futures and forward convenience yield dynamics.

Proposition 3.2 Assume that the dynamics of the futures and forward convenience yields, under the martingale measure $Q$, are given by

$$
d \varphi(t, T)=\alpha_{\varphi}(t, T) d t+\sigma_{\varphi}(t, T) d W_{t}
$$




$$
\begin{aligned}
& +\int_{E} \delta_{\varphi}(t, y, T) \mu(d t, d y), \\
d \gamma(t, T) & =\alpha_{\gamma}(t, T) d t+\sigma_{\gamma}(t, T) d W_{t} \\
& +\int_{E} \delta_{\gamma}(t, y, T) \mu(d t, d y) .
\end{aligned}
$$

Then the futures and forward yield drift terms are given by

$$
\begin{aligned}
\alpha_{\varphi}(t, T) & =\sigma_{f}(t, T) \int_{t}^{T} \sigma_{f}^{\star}(t, s) d s-\int_{E} \delta_{f}(t, x, T) e^{-D_{f}(t, x, T)} \lambda(t, d x) \\
& +\left\{\sigma_{f}(t, T)-\sigma_{\varphi}(t, T)\right\}\left\{\sigma_{S}^{\star}(t)+\int_{t}^{T}\left[\sigma_{f}^{\star}(t, T)-\sigma_{\varphi}^{\star}(t, T)\right] d s\right\} \\
& +\int_{E}\left\{\delta_{f}(t, y, T)-\delta_{\varphi}(t, y, T)\right\}\left\{e^{D_{f}(t, y, T)-D_{\varphi}(t, y, T)}\right\}\left(\delta_{S}(t, y)+1\right) \lambda(t, d y) . \\
\alpha_{\gamma}(t, T) & =\sigma_{\gamma}(t, T) \int_{t}^{T} \sigma_{\gamma}^{\star}(t, s) d s-\sigma_{\gamma}(t, T) \sigma_{S}^{\star}(t) \\
& -\int_{E} \delta_{\gamma}(t, y, T) e^{-D_{\gamma}(t, y, T)}\left(\delta_{S}(t, y)+1\right) \lambda(t, d y) .
\end{aligned}
$$

Proof. We have by definition

$$
F(t, T)=S_{t} e^{Z(t, T)},
$$

with $Z$ defined as

$$
Z(t, T)=\int_{t}^{T}[f(t, s)-\varphi(t, s)] d s .
$$

From Lemma 3.1, together with the facts that $f(t, t)=r_{t}$ and $\varphi(t, t)=c_{t}$ we obtain (suppressing $(t, T)$ )

$$
\begin{aligned}
d Z & =\left\{c_{t}-r_{t}+A_{f}-A_{\varphi}\right\} d t+\left\{S_{f}-S_{\varphi}\right\} d W_{t} \\
& +\int_{E}\left(D_{f}-D_{\varphi}\right)(t, y, T) \mu(d t, d y) .
\end{aligned}
$$

Defining, for each $T$, the process $X(t, T)$ by $X(t, T)=e^{Z(t, T)}$, Itô's formula now gives us (suppressing $T$ )

$$
\begin{aligned}
d X_{t} & =X_{t}\left\{c_{t}-r_{t}+A_{f}-A_{\varphi}+\frac{1}{2}\left\|S_{f}-S_{\varphi}\right\|^{2}\right\} d t+X_{t}\left\{S_{f}-S_{\varphi}\right\} d W_{t} \\
& +X_{t-} \int_{E}\left\{e^{D_{f}-D_{\varphi}}-1\right\} \mu(d t, d y) .
\end{aligned}
$$


From this expression, the spot price dynamics (5) and the relation (22), an application of the Itô formula to the expression $F(t, T)=S_{t} X(t, T)$ gives us

$$
\begin{aligned}
d F_{t} & =F_{t}\left\{c_{t}-r_{t}+A_{f}-A_{\varphi}+\frac{1}{2}\left\|S_{f}-S_{\varphi}\right\|^{2}\right\} d t \\
& +F_{t}\left\{r_{t}-c_{t}-\int_{E} \delta_{S}(t, y) \lambda(t, d y)\right\} d t \\
& +F_{t}\left(S_{f}-S_{\varphi}\right) d W_{t} \\
& +F_{t} \sigma_{S} d W_{t} \\
& +F_{t}\left(S_{f}-S_{\varphi}\right) \sigma_{S}^{\star} d t \\
& +F_{t-} \int_{E}\left\{e^{D_{f}-D_{\varphi}}\left(1+\delta_{S}\right)-1\right\} \mu(d t, d y) .
\end{aligned}
$$

Compensating the point process part and collecting terms give us the semimartingale dynamics

$$
\begin{aligned}
d F_{t} & =F_{t}\left\{A_{f}-A_{\varphi}+\frac{1}{2}\left\|S_{f}-S_{\varphi}\right\|^{2}+\left(S_{f}-S_{\varphi}\right) \sigma_{S}^{\star}\right. \\
& \left.+\int_{E}\left(e^{D_{f}-D_{\varphi}}-1\right)\left(1+\delta_{S}\right) \lambda(t, d y)\right\} d t \\
& +F_{t}\left\{S_{f}-S_{\varphi}+\sigma_{S}\right\} d W_{t} \\
& +F_{t-} \int_{E}\left\{e^{D_{f}-D_{\varphi}}\left(1+\delta_{S}\right)-1\right\} \tilde{\mu}(d t, d y),
\end{aligned}
$$

Since $F(t, T)$ is a $Q$-martingale for each fixed $T$, the $d t$-term must vanish, so the following identity must hold for all $t$ and $T$ with $0 \leq t \leq T$.

$$
\begin{aligned}
0= & A_{f}(t, T)-A_{\varphi}(t, T)+\frac{1}{2}\left\|S_{f}(t, T)-S_{\varphi}(t, T)\right\|^{2}+\left(S_{f}(t, T)-S_{\varphi}(t, T)\right) \sigma_{S}^{\star}(t) \\
& +\int_{E}\left(e^{D_{f}(t, y, T)-D_{\varphi}(t, y, T)}-1\right)\left(1+\delta_{S}(t, y)\right) \lambda(t, d y) .
\end{aligned}
$$

Differentiating this identity w.r.t. $T$, using (17) and rearranging, we obtain the drift condition for $\alpha_{\varphi}$.

In order to derive the forward yield drift condition we obtain, in the same way as for $d F$,

$$
\begin{aligned}
d G_{t} & =G_{t}\left\{A_{f}-A_{\gamma}+\frac{1}{2}\left\|S_{f}-S_{\gamma}\right\|^{2}+\left(S_{f}-S_{\gamma}\right) \sigma_{S}^{\star}-\int_{E} \delta_{S} \lambda(t, d y)\right\} d t \\
& +G_{t}\left\{S_{f}-S_{\gamma}+\sigma_{S}\right\} d W_{t} \\
& +G_{t-} \int_{E}\left\{e^{D_{f}-D_{\gamma}}\left(1+\delta_{S}\right)-1\right\} \mu(d t, d y),
\end{aligned}
$$

Applying Proposition 2.3 to the $G$-dynamics thus derived and taking the $T$ derivative, gives us the result for $\alpha_{\gamma}$. 


\section{Affine term structures for forwards and fu- tures}

Modeling the entire term structure of forward or futures prices results in an infinite dimensional state variable. Therefore it is sometimes more convenient to model a finite dimensional state process $Z$, and to assume that forward and futures prices are given as functions of this state process. Just as in interest rate theory (see [7], [8]), the term structures defined by functions which are exponentially affine in the state variables are computationally very tractable, and below we give necessary and sufficient conditions in terms of the dynamics of the state process $Z$ for the forward and futures term structures to be affine.

Assumption 4.1 The m-dimensional Markov process $Z$ is assumed to have a stochastic differential given by

$$
d Z_{t}=\alpha_{Z}\left(t, Z_{t}\right) d t+\sigma_{Z}\left(t, Z_{t}\right) d W_{t}+\int_{E} \delta_{Z}\left(t, Z_{t-}, y\right) \mu(d t, d y)
$$

under the martingale measure $Q$. Furthermore we assume that the compensator $\nu$ of $\mu$ can be written as $\nu(\omega ; d t, d y)=\lambda\left(t, Z_{t-}(\omega), d y\right) d t$.

\subsection{Futures}

Assumption 4.2 We assume that the futures prices can be written on the following form

$$
F(t, T)=H_{F}\left(t, Z_{t}, T\right),
$$

where $H_{F}: R^{3} \rightarrow R$ is a smooth function. In particular we assume that the spot price $S$ is given by

$$
S(t)=H_{F}\left(t, Z_{t}, t\right)=h\left(t, Z_{t}\right) .
$$

Lemma 4.1 If futures prices are given by (36), then $H_{F}$ satisfies the following partial differential equation

$$
\left\{\begin{aligned}
\frac{\partial H_{F}}{\partial t}(t, z, T)+\mathcal{A} H_{F}(t, z, T) & =0, \\
H_{F}(T, z, T) & =h(T, z),
\end{aligned}\right.
$$

where $\mathcal{A}$ is given by

$$
\begin{aligned}
\mathcal{A} H(t, z, T)= & \sum_{i=1}^{m} \alpha_{Z}^{i}(t, z) \frac{\partial H}{\partial z_{i}}+\frac{1}{2} \sum_{i, j=1}^{m} C_{i j}(t, z) \frac{\partial^{2} H}{\partial z_{i} \partial z_{j}} \\
& +\int_{E}\left[H\left(t, z+\delta_{Z}(t, z, y), T\right)-H(t, z, T)\right] \lambda(t, z, d y) .
\end{aligned}
$$

In the expression (38) the matrix $C$ is defined by

$$
C=\sigma_{Z} \sigma_{Z}^{*}
$$

where * denotes transpose and all the partial derivatives of $H$ should be evaluated at $(t, z, T)$. 
Proof. Dynkin's formula on

$$
H_{F}\left(t, Z_{t}, T\right)=E^{Q}\left[h\left(T, Z_{T}\right) \mid \mathcal{F}_{t}\right] .
$$

Definition 4.1 The term structure of futures prices is said to be affine if the function $H_{F}$ from (36) is of the following form

$$
\ln H_{F}(t, z, T)=A_{F}(t, T)+B_{F}^{*}(t, T) z,
$$

where $A_{F}$ and $B_{F}$ are deterministic functions.

Proposition 4.1 Suppose that Assumption 4.2 is in force and that the functions $\alpha_{Z}, \sigma_{Z}, \delta_{Z}, \lambda$ and $h$ are of the following form

$$
\begin{aligned}
\alpha_{Z}(t, z) & =a_{1}(t)+a_{2}(t) z, \\
\sigma_{Z} \sigma_{Z}^{*}(t, z) & =k_{0}(t)+\sum_{i=1}^{m} k_{i}(t) z_{i}, \\
\delta_{Z}(t, z, y) & =\delta_{Z}(t, y), \\
\lambda(t, z, y) & =l_{1}(t, y)+l_{2}^{*}(t, y) z, \\
\ln h(t, z) & =c(t)+d^{*}(t) z .
\end{aligned}
$$

Then the term structure of futures prices is affine, that is $H_{F}$ from (36) can be written on the form (40) where $A_{F}$ and $B_{F}$ solve the following system of ordinary differential equations.

$$
\left\{\begin{aligned}
\frac{\partial A_{F}(t, T)}{\partial t}+a_{1}^{*}(t) B_{F}(t, T)+\frac{1}{2} B_{F}^{*}(t, T) k_{0}(t) B_{F}(t, T) & \\
+\int_{E}\left(e^{B_{F}^{*}(t, T) \delta_{Z}(t, y)}-1\right) l_{1}(t, d y) & =0 \\
A_{F}(T, T) & =c(T),
\end{aligned}\right.
$$

and

$$
\left\{\begin{aligned}
\frac{\partial B_{F}(t, T)}{\partial t}+a_{2}^{*}(t) B_{F}(t, T)+\frac{1}{2} \beta_{F}^{*}(t, T) K(t) B_{F}(t, T) & \\
+\int_{E}\left(e^{B_{F}^{*}(t, T) \delta_{Z}(t, y)}-1\right) l_{2}(t, d y) & =0 \\
B_{F}(T, T) & =d(T),
\end{aligned}\right.
$$

where

$$
K(t)=\left[\begin{array}{c}
k_{1}(t) \\
k_{2}(t) \\
\vdots \\
k_{m}(t)
\end{array}\right]
$$


and $\beta_{F}(t, T)$ is the $m^{2} \times m$-matrix

$$
\beta_{F}(t, T)=\left[\begin{array}{cccc}
B_{F}(t, T) & 0 & \cdots & 0 \\
0 & B_{F}(t, T) & & \\
\vdots & & \ddots & \\
0 & & & B_{F}(t, T)
\end{array}\right] .
$$

Proof. This follows from the fact that $\exp \left\{A_{F}(t, T)+B_{F}(t, T)\right\}$, where $A_{F}$ and $B_{F}$ solve (42) and (43), respectively, solves the PDE (37), which uniquely characterizes the futures prices in this setting.

Remark 4.1 As in [7] it can be shown that under non degeneracy conditions and a possible reordering of indices a $\sigma_{Z}$ of the form in (41) can be written as

$$
\sigma_{Z}\left(t, Z_{t}\right)=\Sigma(t)\left[\begin{array}{cccc}
\sqrt{u_{1}\left(t, Z_{t}\right)} & 0 & \cdots & 0 \\
0 & \sqrt{u_{2}\left(t, Z_{t}\right)} & \cdots & 0 \\
& & \ddots & \\
0 & \cdots & 0 & \sqrt{u_{m}\left(t, Z_{t}\right)}
\end{array}\right]
$$

where the matrix $\Sigma(t)$ depends only on $t$ and

$$
u_{i}(t, z)=k_{0}^{i}(t)+\sum_{j=1}^{m} k_{j}^{i}(t) z_{j} .
$$

In most models which have appeared in the literature, the spot price is one factor. Note that if we want an affine term structure we can not use the spot price itself as a factor, but we must use the logarithm of the spot price. Also note that if the logarithm of the spot price is a factor, then the boundary conditions of $A_{F}$ and $B_{F}$ will be uniquely determined. We summarize these observations in the following corollary of Proposition 4.1.

Corollary 4.1 Assume that $Z^{0}(t)=\ln S(t)$ and that $\alpha_{Z}, \sigma_{Z} \sigma_{Z}^{*}, \delta_{Z}$ and $\lambda$ are of the form stated in Proposition 4.1. Then

$$
h(t, z)=e^{z^{0}},
$$

and the term structure of futures prices is affine. That is, $H_{F}$ from (36) can be written on the form (40) where $A_{F}$ and $B_{F}$ solve the system (42)-(43) of ordinary differential equations with the boundary conditions replaced by

$$
A_{F}(T, T)=0,
$$

and

$$
B_{F}(T, T)=e_{0},
$$


where $e_{0}=(1,0, \ldots, 0)^{*}$.

The stochastic differential of $Z^{0}$ has the required form if and only if the stochastic differential of the spot price $S$ can be written as

$$
\begin{aligned}
d S_{t}= & {\left[\beta_{1}(t)+\beta_{2}(t) \ln S_{t}+\sum_{i=1}^{m} \beta_{3}^{i}(t) Z_{t}^{i}\right] S_{t} d t } \\
& +\sigma_{S}\left(t, Z_{t}\right) S_{t} d W_{t} \\
& +S_{t-} \int_{E} \delta_{S}(t, y) \mu(d t, d y),
\end{aligned}
$$

where

$$
\sigma_{S}\left(t, Z_{t}\right) \sigma_{S}^{*}\left(t, Z_{t}\right)=\Gamma_{1}(t)+\Gamma_{2}(t) \ln S_{t}+\sum_{i=1}^{m} \Gamma_{3}(t) Z_{t}^{i}
$$

\subsection{Examples}

A number of factor models have been proposed in the literature, and the corresponding futures prices have been computed on a case by case basis. We now give a list of the most well known factor models and it follows immediately from Corollary 4.1 that all these models will give rise to an affine term structure of futures prices, which thus easily can be computed. We also provide some new examples of affine factor models. All the models are given directly under an equivalent martingale measure $Q$, and unless indicated otherwise, the coefficients of the models are assumed to be constant.

\subsubsection{The Schwartz spot price model.}

The following spot price model was studied in [18].

$$
d S_{t}=\kappa\left(\alpha_{S}-\ln S_{t}\right) S_{t} d t+\sigma_{S} S_{t} d W_{t} .
$$

Here $W$ is a one-dimensional Wiener process. Since this model is a special case of the model below we defer computing the futures prices for this model to the next section.

\subsubsection{The general affine Wiener driven spot price model.}

Without loosing the affine term structure, we can extend the one-factor model by Schwartz to include a volatility of Cox-Ingersoll-Ross type. The model then looks as follows

$$
d S_{t}=\left[a_{1}(t)+a_{2}(t) \ln S_{t}\right] S_{t} d t+S_{t} \sqrt{k_{0}(t)+k_{1}(t) \ln S_{t}} d W_{t} .
$$

The functions $A_{F}$ and $B_{F}$ for this model satisfy the following ordinary differential equations $\left(Z_{t}=\ln S_{t}\right.$ is used)

$$
\begin{aligned}
\frac{\partial A_{F}(t, T)}{\partial t}+\left(a_{1}(t)-\frac{1}{2} k_{0}(t)\right) B_{F}(t, T)+\frac{1}{2} k_{0}(t) B_{F}^{2}(t, T) & =0, \\
A_{F}(T, T) & =0,
\end{aligned}
$$


and

$$
\begin{aligned}
\frac{\partial B_{F}(t, T)}{\partial t}+\left(a_{2}(t)-\frac{1}{2} k_{1}(t)\right) B_{F}(t, T)+\frac{1}{2} k_{1}(t) B_{F}^{2}(t, T) & =0, \\
B_{F}(T, T) & =1 .
\end{aligned}
$$

The solutions are given by

$$
B_{F}(t, T)=\frac{1}{X(t, T)}
$$

where

$$
X(t, T)=e^{-\int_{t}^{T}\left[a_{2}(s)-\frac{1}{2} k_{1}(s)\right] d s}-\frac{1}{2} \int_{t}^{T} e^{-\int_{t}^{u}\left[a_{2}(s)-\frac{1}{2} k_{1}(s)\right] d s} k_{1}(u) d u,
$$

and

$$
A_{F}(t, T)=\int_{t}^{T}\left[\left(a_{1}(s)-\frac{1}{2} k_{0}(s)\right) B_{F}(s, T)+\frac{1}{2} k_{0}(s) B_{F}^{2}(s, T)\right] d s .
$$

As a special case, inserting

$$
\begin{aligned}
& a_{1}(t)=\kappa \alpha_{S}, \\
& a_{2}(t)=-\kappa, \\
& k_{0}(t)=\sigma^{2}, \\
& k_{1}(t)=0,
\end{aligned}
$$

we find the futures prices for the Schwartz one-factor model (see [18]).

\subsubsection{The Gibson-Schwartz two-factor model.}

The following two-factor model uses the spot price and the spot convenience yield as factors. It is based on the model in [10] and appears in [18].

$$
\begin{aligned}
d S_{t} & =\left(r-c_{t}\right) S_{t} d t+S_{t} \sigma_{S} d W_{t}, \\
d c_{t} & =\kappa\left(\alpha_{c}-c_{t}\right) d t+\sigma_{c} d W_{t} .
\end{aligned}
$$

Here $W$ is a two-dimensional Wiener process and

$$
\sigma_{S} \sigma_{c}^{*}=\rho\left\|\sigma_{S}\right\| \cdot\left\|\sigma_{c}\right\| .
$$

Again, this model is a special case of the next and therefore we defer computing the futures prices for this model to the next paragraph.

Note that for the one and two-factor models presented so far, forward and futures prices agree, since interest rates are assumed to be deterministic. 


\subsubsection{The Schwartz three-factor model.}

Including the short rate $r$ as a third factor makes forward and futures prices different. The following model can be found in [18].

$$
\begin{aligned}
d S_{t} & =\left(r_{t}-c_{t}\right) S_{t} d t+S_{t} \sigma_{S} d W_{t}, \\
d c_{t} & =\kappa_{c}\left(\alpha_{c}-c_{t}\right) d t+\sigma_{c} d W_{t}, \\
d r_{t} & =\kappa_{r}\left(\alpha_{r}-r_{t}\right) d t+\sigma_{r} d W_{t},
\end{aligned}
$$

where $W$ is a three-dimensional Wiener process, and

$$
\begin{array}{r}
\sigma_{S} \sigma_{c}^{*}=\rho_{S c}\left\|\sigma_{S}\right\| \cdot\left\|\sigma_{c}\right\|, \\
\sigma_{c} \sigma_{r}^{*}=\rho_{c r}\left\|\sigma_{c}\right\| \cdot\left\|\sigma_{r}\right\|, \\
\sigma_{S} \sigma_{r}^{*}=\rho_{S r}\left\|\sigma_{S}\right\| \cdot\left\|\sigma_{r}\right\|,
\end{array}
$$

Let $Z_{t}=\left[\ln S_{t}, c_{t}, r_{t}\right]^{*}$. The functions $A_{F}$ and $B_{F}=\left[B_{S}, B_{c}, B_{r}\right]^{*}$ for this model satisfy the following ordinary differential equations

$$
\begin{aligned}
\frac{\partial A_{F}(t, T)}{\partial t}-\frac{1}{2}\left\|\sigma_{S}\right\|^{2} B_{S}(t, T)+\kappa_{c} \alpha_{c} B_{c}(t, T)+\kappa_{r} \alpha_{r} B_{r}(t, T) & \\
+\frac{1}{2} B_{F}(t, T)^{*} k_{0} B_{F}(t, T) & =0, \\
A_{F}(T, T) & =0,
\end{aligned}
$$

where $k_{0}$ is the covariance matrix

$$
k_{0}=\left[\begin{array}{ccc}
\left\|\sigma_{S}\right\|^{2} & \rho_{S c}\left\|\sigma_{S}\right\| \cdot\left\|\sigma_{c}\right\| & \rho_{S r}\left\|\sigma_{S}\right\| \cdot\left\|\sigma_{r}\right\| \\
\rho_{S c}\left\|\sigma_{S}\right\| \cdot\left\|\sigma_{c}\right\| & \left\|\sigma_{c}\right\|^{2} & \rho_{c r}\left\|\sigma_{c}\right\| \cdot\left\|\sigma_{r}\right\| \\
\rho_{S r}\left\|\sigma_{S}\right\| \cdot\left\|\sigma_{r}\right\| & \rho_{c r}\left\|\sigma_{c}\right\| \cdot\left\|\sigma_{r}\right\| & \left\|\sigma_{r}\right\|^{2}
\end{array}\right],
$$

and

$$
\begin{aligned}
\frac{\partial B_{F}(t, T)}{\partial t}+a_{2}^{*} B_{F}(t, T) & =0 \\
B_{F}(T, T) & =[1,0,0]^{*}
\end{aligned}
$$

where the matrix $a_{2}$ is given by

$$
a_{2}=\left[\begin{array}{ccc}
0 & -1 & 1 \\
0 & -\kappa_{c} & 0 \\
0 & 0 & -\kappa_{r}
\end{array}\right]
$$

The solutions are given by

$$
\begin{aligned}
B_{S}(t, T) & \equiv 1, \\
B_{c}(t, T) & =\frac{1}{\kappa_{c}}\left(e^{-\kappa_{c}(T-t)}-1\right), \\
B_{r}(t, T) & =\frac{1}{\kappa_{r}}\left(1-e^{-\kappa_{r}(T-t)}\right),
\end{aligned}
$$


and

$$
\begin{aligned}
A_{F}(t, T)= & \frac{\kappa_{c} \alpha_{c}+\rho_{S c}\left\|\sigma_{S}\right\| \cdot\left\|\sigma_{c}\right\|}{\kappa_{c}^{2}}\left(1-e^{-\kappa_{c}(T-t)}-\kappa_{c}(T-t)\right) \\
& -\frac{\kappa_{r} \alpha_{r}+\rho_{S r}\left\|\sigma_{S}\right\| \cdot\left\|\sigma_{r}\right\|}{\kappa_{r}^{2}}\left(1-e^{-\kappa_{r}(T-t)}-\kappa_{r}(T-t)\right) \\
& +\frac{\left\|\sigma_{c}\right\|^{2}}{4 \kappa_{c}^{3}}\left(2 \kappa_{c}(T-t)-3+4 e^{-\kappa_{c}(T-t)}-e^{-2 \kappa_{c}(T-t)}\right) \\
& +\frac{\left\|\sigma_{r}\right\|^{2}}{4 \kappa_{r}^{3}}\left(2 \kappa_{r}(T-t)-3+4 e^{-\kappa_{r}(T-t)}-e^{-2 \kappa_{r}(T-t)}\right) \\
& +\frac{\rho_{c r}\left\|\sigma_{c}\right\| \cdot\left\|\sigma_{r}\right\|}{\kappa_{c}+\kappa_{r}}\left\{\frac{1-e^{-\kappa_{c}(T-t)}-e^{-\kappa_{r}(T-t)}+e^{-\left(\kappa_{c}+\kappa_{r}\right)(T-t)}}{\kappa_{c} \kappa_{r}}\right. \\
& \left.+\frac{1}{\kappa_{c}^{2}}\left(1-e^{-\kappa_{c}(T-t)}-\kappa_{c}(T-t)\right)+\frac{1}{\kappa_{r}^{2}}\left(1-e^{-\kappa_{r}(T-t)}-\kappa_{r}(T-t)\right)\right\} .
\end{aligned}
$$

Remark 4.2 Using the explicit expression for bond prices for this model (see [19]) we see that the futures prices can be written in the following manner

$$
F(t, T)=\frac{S_{t}}{p(t, T)} e^{B_{c}(t, T) c_{t}} e^{\bar{A}(t, T)}
$$

where

$$
\bar{A}(t, T)=A_{F}(t, T)+\frac{\left\|\sigma_{r}\right\|^{2}}{4 \kappa_{r}^{3}}\left(2 \kappa_{r}(T-t)-3+4 e^{-\kappa_{r}(T-t)}-e^{-2 \kappa_{r}(T-t)}\right)
$$

This result will hold even if $\alpha_{r}$ is allowed to be time-dependent.

\subsubsection{The Hilliard-Reis three-factor model.}

This model was suggested in [11], and coincides with the Schwartz three-factor model except for the facts that the spot price process includes jumps and that the drift of the short rate is time dependent. The function $\alpha_{r}(t)$ below is chosen so that the initial bond prices produced by the model agree with the observed bond prices. The model is defined by

$$
\begin{aligned}
d S_{t} & =\left(r_{t}-c_{t}\right) S_{t} d t+S_{t} \sigma_{S} d W_{t}+S_{t-} \int_{R} y \tilde{\mu}(d t, d y), \\
d c_{t} & =\kappa_{c}\left(\alpha_{c}-c_{t}\right) d t+\sigma_{c} d W_{t}, \\
d r_{t} & =\kappa_{r}\left(\alpha_{r}(t)-r\right) d t+\sigma_{r} d W_{t},
\end{aligned}
$$

where $W$ and $\sigma_{S}, \sigma_{c}$ and $\sigma_{r}$ are as in Section 4.2.4, and as before $\tilde{\mu}$ is given by $\tilde{\mu}(d t, d y)=\mu(d t, d y)-\nu(d t, d y)$. Here the marked point process $\mu$ has mark space $(R, \mathcal{B})$, where $\mathcal{B}$ is the Borel algebra, and the compensator $\nu$ is given by

$$
\nu(d t, d y)=\lambda d t \frac{1}{\sqrt{2 \pi \eta^{2}}} \cdot \frac{1}{y+1} \exp \left\{-\frac{[\ln (y+1)-\xi]^{2}}{2 \eta^{2}}\right\} d y,
$$


for $-1<y<\infty$. This means that the spot price process will jump according to a Poisson process with intensity $\lambda$ and that if $\delta$ denotes the relative jump size, then $1+\delta$ has a log-normal distribution: $\ln (1+\delta) \sim N\left(\xi, \eta^{2}\right)$.

Since the spot price for this model equals the spot price for the Schwartz three-factor model (with a time dependent $\alpha_{r}$, see Remark 4.2) plus a $Q$ martingale, the futures prices will be the same for these models (as was pointed out in [11]).

\subsubsection{A three-factor model with positive short rate}

If we want to be sure that the short rate is positive, we could replace the short rate process assumed in the models above by a Cox-Ingersoll-Ross type process. With a wise choice of the parameters $\kappa_{r}, \alpha_{r}$ and $\sigma_{r}$ the following model will have a positive short rate (see [6]). This model has (to our knowledge) not been studied previously.

$$
\begin{aligned}
d S_{t} & =\left(r_{t}-c_{t}\right) S_{t} d t+S_{t} \sigma_{S} d W_{t}, \\
d c_{t} & =\kappa_{c}\left(\alpha_{c}-c_{t}\right) d t+\sigma_{c} d W_{t}, \\
d r_{t} & =\kappa_{r}\left(\alpha_{r}-r_{t}\right) d t+\sqrt{r_{t}} \sigma_{r} d W_{t} .
\end{aligned}
$$

Again $W$ is assumed to be a three-dimensional Wiener process, and

$$
\begin{aligned}
\sigma_{S} \sigma_{c}^{*} & =\rho_{S c}\left\|\sigma_{S}\right\| \cdot\left\|\sigma_{c}\right\|, \\
\sigma_{c} \sigma_{r}^{*} & =0 \\
\sigma_{S} \sigma_{r}^{*} & =0
\end{aligned}
$$

Let $Z_{t}=\left[\ln S_{t}, c_{t}, r_{t}\right]^{*}$. The functions $A_{F}$ and $B_{F}=\left[B_{S}, B_{c}, B_{r}\right]^{*}$ for this model satisfy the following ordinary differential equations

$$
\begin{aligned}
\frac{\partial A_{F}(t, T)}{\partial t}-\frac{1}{2}\left\|\sigma_{S}\right\|^{2} B_{S}(t, T)+\kappa_{c} \alpha_{c} B_{c}(t, T)+\kappa_{r} \alpha_{r} B_{r}(t, T) & \\
+\frac{1}{2} B_{F}(t, T)^{*} k_{0} B_{F}(t, T) & =0 \\
A_{F}(T, T) & =0
\end{aligned}
$$

where $k_{0}$ is the matrix

$$
k_{0}=\left[\begin{array}{ccc}
\left\|\sigma_{S}\right\|^{2} & \rho_{S c}\left\|\sigma_{S}\right\| \cdot\left\|\sigma_{c}\right\| & 0 \\
\rho_{S c}\left\|\sigma_{S}\right\| \cdot\left\|\sigma_{c}\right\| & \left\|\sigma_{c}\right\|^{2} & 0 \\
0 & 0 & 0
\end{array}\right],
$$

and

$$
\begin{aligned}
\frac{\partial B_{F}(t, T)}{\partial t}+a_{2}^{*} B_{F}(t, T)+\frac{1}{2} B_{F}^{*}(t, T) k_{r} B_{F}(t, T) & =0, \\
B_{F}(T, T) & =[1,0,0]^{*},
\end{aligned}
$$


where the matrix $a_{2}$ is given by

$$
a_{2}=\left[\begin{array}{ccc}
0 & -1 & 1 \\
0 & -\kappa_{c} & 0 \\
0 & 0 & -\kappa_{r}
\end{array}\right],
$$

and the matrix $k_{r}$ by

$$
k_{r}=\left[\begin{array}{ccc}
0 & 0 & 0 \\
0 & 0 & 0 \\
0 & 0 & \left\|\sigma_{r}\right\|^{2}
\end{array}\right] .
$$

The solutions are given by

$$
\begin{aligned}
B_{S}(t, T) & \equiv 1 \\
B_{c}(t, T) & =\frac{1}{\kappa_{c}}\left(e^{-\kappa_{c}(T-t)}-1\right) \\
B_{r}(t, T) & =-\frac{2}{\left\|\sigma_{r}\right\|^{2}} \cdot \frac{\left(2 r_{2}+\left\|\sigma_{r}\right\|^{2}\right) r_{1} e^{-r_{1}(T-t)}-\left(2 r_{1}+\left\|\sigma_{r}\right\|^{2}\right) r_{2} e^{-r_{2}(T-t)}}{\left(2 r_{2}+\left\|\sigma_{r}\right\|^{2}\right) e^{-r_{1}(T-t)}-\left(2 r_{1}+\left\|\sigma_{r}\right\|^{2}\right) e^{-r_{2}(T-t)}}
\end{aligned}
$$

where

$$
\begin{aligned}
& r_{1}=\frac{\kappa_{r}}{2}+\sqrt{\frac{\kappa_{r}^{2}}{4}+\frac{\left\|\sigma_{r}\right\|^{2}}{2}} \\
& r_{2}=\frac{\kappa_{r}}{2}-\sqrt{\frac{\kappa_{r}^{2}}{4}+\frac{\left\|\sigma_{r}\right\|^{2}}{2}}
\end{aligned}
$$

Once $B_{F}$ has been determined $A_{F}$ can obtained via numerical integration.

\subsection{Forwards}

Assumption 4.3 We assume that the zero-coupon bond prices are of the form

$$
p(t, T)=H_{P}\left(t, Z_{t}, T\right),
$$

where $H_{P}: R^{3} \rightarrow R$ is a smooth function. Furthermore, we assume that the forward prices can be written on the following form

$$
G(t, T)=H_{G}\left(t, Z_{t}, T\right),
$$

where $H_{G}: R^{3} \rightarrow R$ is a smooth function. In particular we assume that the spot price $S$ is given by

$$
S(t)=H_{G}\left(t, Z_{t}, t\right)=h\left(t, Z_{t}\right) .
$$

Lemma 4.2 If zero-coupon bond prices are given by (51) and forward prices are given by (52), then $H_{G}$ satisfies the following partial differential equation

$$
\left\{\begin{aligned}
\frac{\partial H_{G}}{\partial t}(t, z, T)+\mathcal{A}^{T} H_{G}(t, z, T) & =0, \\
H_{G}(T, z, T) & =h(T, z),
\end{aligned}\right.
$$


where $\mathcal{A}^{T}$ is given by

$$
\begin{aligned}
\mathcal{A}^{T} H(t, z, T) & =\sum_{i=1}^{m}\left(\alpha_{Z}^{T}\right)^{i}(t, z) \frac{\partial H}{\partial z_{i}}+\frac{1}{2} \sum_{i, j=1}^{m} C_{i j}(t, z) \frac{\partial^{2} H}{\partial z_{i} \partial z_{j}} \\
& +\int_{E}\left[H\left(t, z+\delta_{Z}(t, z, y), T\right)-H(t, z, T)\right] \lambda^{T}(t, z, d y) .
\end{aligned}
$$

Here

$$
\begin{aligned}
\alpha_{Z}^{T}(t, z) & =\alpha_{Z}(t, z)+\sigma_{Z}(t, z) \sigma_{P}^{*}(t, z, T), \\
\lambda^{T}(t, z, d y) & =\left[\delta_{P}(t, z, y, T)+1\right] \lambda(t, z, d y), \\
\sigma_{P}(t, z, T) & =\frac{\nabla_{z} H_{P}(t, z, T)}{H_{P}(t, z, T)} \sigma_{Z}(t, z), \\
\delta_{P}(t, z, y, T) & =\frac{H_{P}\left(t, z+\delta_{Z}(t, z, y), T\right)-H_{P}(t, z, T)}{H_{P}(t, z, T)} .
\end{aligned}
$$

The matrix $C$ was defined in (39). In the expression (54) all the partial derivatives of $H$ should be evaluated at $(t, z, T)$.

Proof. Itô's formula applied to $p(t, T)=H_{P}\left(t, Z_{t}, T\right)$ gives the expressions for $\sigma_{P}$ and $\delta_{P}$. The dynamics of $Z$ under the $T$-forward measure $Q^{T}$ can be found using (18) and (19) and they are given by

$$
d Z_{t}=\alpha_{Z}^{T}\left(t, Z_{t}\right) d t+\sigma_{Z}\left(t, Z_{t}\right) d W_{t}^{T}+\int_{E} \delta_{Z}\left(t, Z_{t-}, y\right) \mu(d t, d y)
$$

where the intensity of $\mu$ is $\lambda^{T}\left(t, Z_{t-}(\omega), d y\right)$. The result now follows from an application of Dynkin's formula to

$$
H_{G}\left(t, Z_{t}, T\right)=E^{T}\left[h\left(T, Z_{T}\right) \mid \mathcal{F}_{t}\right] .
$$

Definition 4.2 The term structure of interest rates is said to be affine if the function $H_{P}$ from (51) is of the following form

$$
\ln H_{P}(t, z, T)=A_{P}(t, T)+B_{P}^{*}(t, T) z,
$$

where $A_{P}$ and $B_{P}$ are deterministic functions. Analogously, the term structure of forward prices is said to be affine if the function $H_{G}$ from (52) is of the form

$$
\ln H_{G}(t, z, T)=A_{G}(t, T)+B_{G}^{*}(t, T) z,
$$

where $A_{G}$ and $B_{G}$ are deterministic functions. 
Proposition 4.2 Suppose that Assumption 4.3 is in force. Furthermore, suppose that the term structure of interest rates is affine, that is the function $H_{P}$ from (51) can be written on the form (56) and that the functions $\alpha_{Z}, \sigma_{Z}, \delta_{Z}, \lambda$ and $h$ are of the form given in (41). Then the term structure of forward prices is affine, that is $H_{F}$ from (52) can be written on the form (57) where $A_{G}$ and $B_{G}$ solve the following system of ordinary differential equations.

$$
\left\{\begin{aligned}
\frac{\partial A_{G}(t, T)}{\partial t} & +B_{G}^{*}(t, T)\left[a_{1}(t)+k_{0}(t) B_{P}(t, T)\right] \\
& +\frac{1}{2} B_{G}^{*}(t, T) s_{0}(t) B_{G}(t, T) \\
& +\int_{E}\left(e^{B_{G}^{*}(t, T) \delta_{Z}(t, y)}-1\right) e^{B_{P}^{*}(t, T) \delta_{Z}(t, y)} l_{1}(t, d y)=0, \\
A_{G}(T, T) & =c(T)
\end{aligned}\right.
$$

where $K$ is given by (44) and

$$
\beta_{G}(t, T)=\left[\begin{array}{cccc}
B_{G}(t, T) & 0 & \cdots & 0 \\
0 & B_{G}(t, T) & & \\
\vdots & & \ddots & \\
0 & & & B_{G}(t, T)
\end{array}\right] .
$$

Proof. This follows from the fact that $\exp \left\{A_{G}(t, T)+B_{G}(t, T)\right\}$, where $A_{G}$ and $B_{G}$ solve (58) and (59), respectively, solves the PDE (53), which uniquely characterizes the forward prices in this setting.

\section{Options on futures prices}

\subsection{General formula}

In this section we will consider pricing options on futures. To obtain pricing formulas we will use the change of numeraire technique developed in [9]. As before we let $Q$ denote the martingale measure. Apart from $Q$ the measures 
$Q^{T}, Q^{F_{T}}, Q^{F}$ and $Q^{T_{F}}$ will appear. They are defined as follows, starting with the measure $Q^{F}$

$$
d Q^{F}=L_{t}^{F} d Q, \quad \text { on } \mathcal{F}_{t}
$$

where

$$
L_{t}^{F}=\frac{F\left(t, T_{1}\right)}{F\left(0, T_{1}\right)} .
$$

Using Itô's formula the dynamics of $L^{F}$ are obtained as

$$
d L_{t}^{F}=L_{t}^{F} \sigma_{F}\left(t, T_{1}\right) d W_{t}+L_{t-}^{F} \int_{E} \delta_{F}\left(t, y, T_{1}\right) \tilde{\mu}(d t, d y) .
$$

Now, the measure $Q^{T_{F}}$ is defined by

$$
d Q^{T_{F}}=R^{T_{F}} d Q^{F}, \quad \text { on } \mathcal{F}_{T}
$$

where

$$
R^{T_{F}}=\frac{\exp \left\{-\int_{0}^{T} r_{s} d s\right\}}{E^{F}\left[\exp \left\{-\int_{0}^{T} r_{s} d s\right\}\right]} .
$$

Here the super index $F$ indicates that the expectation should be taken under $Q^{F}$.

As we have seen before the $T$-forward measure $Q^{T}$ is defined by

$$
d Q^{T}=L_{t}^{T} d Q, \quad \text { on } \mathcal{F}_{t},
$$

where

$$
L_{t}^{T}=\frac{p(t, T)}{B(t) p(0, T)}
$$

Using Itô's formula the dynamics of $L^{T}$ are obtained as

$$
d L_{t}^{T}=L_{t}^{T} \sigma_{p}(t, T) d W_{t}+L_{t-}^{T} \int_{E} \delta_{p}(t, y, T) \tilde{\mu}(d t, d y) .
$$

Finally, the measure $Q^{F_{T}}$ is defined by

$$
d Q^{F_{T}}=L_{t}^{F_{T}} d Q^{T}, \quad \text { on } \mathcal{F}_{t},
$$

where

$$
\begin{aligned}
L_{t}^{F_{T}}= & \exp \left\{-\int_{0}^{t}\left[\sigma_{F}\left(u, T_{1}\right) \sigma_{p}^{*}(u, T)\right.\right. \\
& \left.\left.+\int_{E} \delta_{F}\left(u, y, T_{1}\right) \delta_{p}(u, y, T) \lambda(u, d y)\right] d u\right\} \frac{F\left(t, T_{1}\right)}{F\left(0, T_{1}\right)} .
\end{aligned}
$$

The $Q^{T}$-dynamics of $F\left(t, T_{1}\right)$ can be found using the Girsanov Theorem. Itô's formula then gives us the dynamics of $L^{F_{T}}$ under $Q^{T}$

$$
d L_{t}^{F_{T}}=L_{t}^{F_{T}} \sigma_{F}\left(t, T_{1}\right) d W_{t}^{T}+L_{t-}^{F_{T}} \int_{E} \delta_{F}\left(t, y, T_{1}\right) \tilde{\mu}(d t, d y) .
$$


The point process $\mu$ has the intensity $\lambda^{T}$ under $Q^{T}$, where $\lambda^{T}(t, d y)$ is given by $\lambda^{T}(t, d y)=\left(1+\delta_{p}(t, y, T)\right) \lambda(t, d y)$.

We may now state the main result of this section

Theorem 5.1 The price, at date zero, of a European call option with exercise date $T$ and exercise price $K$ written on the futures price with delivery date $T_{1}$ can be computed from either one of the following two formulas

$$
\begin{aligned}
C_{F}= & F\left(0, T_{1}\right) E^{F}\left[e^{-\int_{0}^{T} r(s) d s}\right] Q^{T_{F}}\left(F\left(T, T_{1}\right) \geq K\right) \\
& -K p(0, T) Q^{T}\left(F\left(T, T_{1}\right) \geq K\right),
\end{aligned}
$$

where the super index $F$ indicates that the expectation should be taken under $Q^{F}$, or

$$
\begin{aligned}
C_{F}= & p(0, T) F\left(0, T_{1}\right) E^{F_{T}}\left[\exp \left\{\int_{0}^{T} \kappa_{s} d s\right\} I\left\{F\left(T, T_{1}\right) \geq K\right\}\right] \\
& -K p(0, T) Q^{T}\left(F\left(T, T_{1}\right) \geq K\right),
\end{aligned}
$$

where the super index $F_{T}$ indicates that the expectation should be taken under $Q^{F_{T}}$, and $\kappa$ is given by

$$
\kappa(t)=\sigma_{F}\left(t, T_{1}\right) \sigma_{p}^{*}(t, T)+\int_{E} \delta_{F}\left(t, y, T_{1}\right) \delta_{p}(t, y, T) \lambda(t, d y) .
$$

Proof. Write the option as

$$
X=\max \left\{F\left(T, T_{1}\right)-K, 0\right\}=\left[F\left(T, T_{1}\right)-K\right] \cdot I\left\{F\left(T, T_{1}\right) \geq K\right\},
$$

where $I$ is the indicator function, i.e.

$$
I\left\{F\left(T, T_{1}\right) \geq K\right\}= \begin{cases}1 & \text { if } F\left(T, T_{1}\right) \geq K \\ 0 & \text { if } F\left(T, T_{1}\right)<K\end{cases}
$$

We then have that the price of the option, at date zero, is given by

$$
\begin{aligned}
C_{F}= & E^{Q}\left[B(T)^{-1}\left[F\left(T, T_{1}\right)-K\right] \cdot I\left\{F\left(T, T_{1}\right) \geq K\right\}\right] \\
= & E^{Q}\left[\exp \left\{-\int_{0}^{T} r_{s} d s\right\} F\left(T, T_{1}\right) \cdot I\left\{F\left(T, T_{1}\right) \geq K\right\}\right] \\
& -K E^{Q}\left[\exp \left\{-\int_{0}^{T} r_{s} d s\right\} \cdot I\left\{F\left(T, T_{1}\right) \geq K\right\}\right]
\end{aligned}
$$

To prove the first formula we change to the measure $Q^{F}$ in first term to obtain

$$
\begin{aligned}
& E^{Q}\left[\exp \left\{-\int_{0}^{T} r_{s} d s\right\} F\left(T, T_{1}\right) \cdot I\left\{F\left(T, T_{1}\right) \geq K\right\}\right] \\
& =F\left(0, T_{1}\right) E^{F}\left[\exp \left\{-\int_{0}^{T} r_{s} d s\right\} \cdot I\left\{F\left(T, T_{1}\right) \geq K\right\}\right],
\end{aligned}
$$


and then we change to the measure $Q^{T_{F}}$. Note that it is the $T$-forward measure as seen from $Q^{F}$.

For the second term we use the $T$-forward measure directly.

To prove the second formula we use the $T$-forward measure $Q^{T}$ in first term to obtain

$$
\begin{aligned}
& E^{Q}\left[\exp \left\{-\int_{0}^{T} r_{s} d s\right\} F\left(T, T_{1}\right) \cdot I\left\{F\left(T, T_{1}\right) \geq K\right\}\right] \\
& =p(0, T) E^{T}\left[F\left(T, T_{1}\right) I\left\{F\left(T, T_{1}\right) \geq K\right\}\right] \\
& =p(0, T) F\left(0, T_{1}\right) E^{T}\left[e^{-\int_{0}^{T} \kappa_{s} d s} \frac{F\left(T, T_{1}\right)}{F\left(0, T_{1}\right)} \cdot e^{\int_{0}^{T} \kappa_{s} d s} I\left\{F\left(T, T_{1}\right) \geq K\right\}\right]
\end{aligned}
$$

and then we change to the measure $Q^{F_{T}}$.

For the second term, again, we use the $T$-forward measure directly.

We may now ask ourselves whether it is possible to interpret the above measures as martingale measures for some numeraire asset. This question is answered by the following lemma, the proof of which is easy and therefore omitted.

\section{Lemma 5.1}

I: Under $Q^{F}$ the process $V^{F}(t)$ defined by $V^{F}(t)=B(t) F\left(t, T_{1}\right)$ acts as a numeraire asset. Its $Q$-dynamics are given by

$$
\begin{aligned}
d V^{F}(t) & =r(t) V^{F}(t) d t+V^{F}(t) \sigma_{F}\left(t, T_{1}\right) d W(t) \\
& +V^{F}(t-) \int_{E} \delta_{F}\left(t, y, T_{1}\right) \tilde{\mu}(d t, d y)
\end{aligned}
$$

The process is the value process of a self-financing portfolio consisting of $B(t)$ $T_{1}$-futures options and $F\left(t, T_{1}\right)$ units of the money account (i.e. $F\left(t, T_{1}\right) / B(t)$ dollars invested in the money account).

II: Under $Q^{F_{T}}$ the process $V^{F_{T}}(t)=e^{-\int_{0}^{t} \kappa_{s} d s} p(t, T) F\left(t, T_{1}\right)$ acts as a $n u$ meraire asset. Here $\kappa$ is the process given by

$$
\kappa(t)=\sigma_{F}\left(t, T_{1}\right) \sigma_{p}^{*}(t, T)+\int_{E} \delta_{F}\left(t, y, T_{1}\right) \delta_{p}(t, y, T) \lambda(t, d y) .
$$

The Q-dynamics of $V^{F_{T}}$ are given by

$$
\begin{aligned}
\frac{d V^{F_{T}}(t)}{V^{F_{T}}(t-)} & =r(t) d t+\left[\sigma_{p}(t, T)+\sigma_{F}\left(t, T_{1}\right)\right] d W(t) \\
& +\int_{E}\left\{\delta_{F}\left(t, y, T_{1}\right)\left[1+\delta_{p}(t, y, T)\right]+\delta_{p}(t, y, T)\right\} \tilde{\mu}(d t, d y) .
\end{aligned}
$$


The process $V^{F_{T}}$ is the value process of a self-financing portfolio consisting of $\exp \left\{-\int_{0}^{t} \kappa_{s} d s\right\} \cdot F\left(t-, T_{1}\right)$ bonds with maturity $T, \exp \left\{-\int_{0}^{t} \kappa_{s} d s\right\} p(t-, T)$ units of $T_{1}$-futures options, and $V(t-)$ units of an asset with price process $\Pi(t) \equiv$ 0 and cumulative dividend process $D$ defined by

$$
\left.d D(t)=\int_{E} \delta_{p}(t, y, T) \delta_{F}\left(t, y, T_{1}\right)\right][\mu(d t, d y)-\lambda(t, d y) d t] .
$$

\section{$5.2 \quad$ Special cases}

\subsubsection{Deterministic volatilities}

Consider the following model under the martingale measure $Q$.

$$
\begin{aligned}
d f(t, T)= & \alpha_{f}(t, T) d t+\sigma_{f}(t, T) d W_{t}+\int_{E} \delta_{f}(t, y, T) \mu(d t, d y) \\
d F(t, T)= & F(t, T) \alpha_{F}(t, T) d t+F(t, T) \sigma_{F}(t, T) d W_{t} \\
& +F(t-, T) \int_{E} \delta_{F}(t, y, T) \mu(d t, d y) .
\end{aligned}
$$

Here $\sigma_{f}$ and $\sigma_{F}$ are assumed to be deterministic functions of the time parameters. Also $\delta_{f}$ and $\delta_{F}$ are assumed to be deterministic functions, now of the time parameters and the mark space variable $y$. Finally, $\alpha_{f}$ and $\alpha_{F}$ are given by the drift conditions (17) and (15), respectively.

Remark 5.1 Instead of modeling the futures prices directly we could model the futures convenience yield and the spot price dynamics. Suppose we do this as

$$
\begin{aligned}
d \varphi(t, T) & =\alpha_{\varphi}(t, T) d t+\sigma_{\varphi}(t, T) d W_{t}+\int_{E} \delta_{\varphi}(t, y, T) \mu(d t, d y), \\
d S_{t} & =\alpha_{S}(t) S_{t} d t+S(t) \sigma_{S}(t) d W_{t}+S_{t-} \int_{E} \delta_{S}(t, y) \mu(d t, d y),
\end{aligned}
$$

where $\sigma_{\varphi}$ and $\sigma_{S}$ are assumed to be deterministic functions of the time parameters, and $\delta_{\varphi}$ and $\delta_{S}$ are assumed to be deterministic functions of the time parameters and the mark space variable $y$, and, finally, $\alpha_{\varphi}$ is given by the drift condition (34), whereas $\alpha_{S}$ is given by (22). Then it is easily seen from (20) that the futures prices resulting from these specifications will have deterministic volatilities, i.e. they will be of the form considered above.

Using the second pricing formula, (64), we see that the price, at date zero, of a European call option with exercise date $T$ and exercise price $K$ written on the futures price with delivery date $T_{1}$ is given by

$$
\begin{aligned}
C_{F}= & p(0, T) F\left(0, T_{1}\right) e^{\int_{0}^{T} \kappa_{s} d s} Q^{F_{T}}\left(F\left(T, T_{1}\right) \geq K\right) \\
& -K p(0, T) Q^{T}\left(F\left(T, T_{1}\right) \geq K\right),
\end{aligned}
$$


since the process $\kappa$ defined in (65) is deterministic for this model (recall formula (11)). The dynamics of $F\left(t, T_{1}\right)$ under $Q^{T}$ and $Q^{F_{T}}$ are easily found using the dynamics of $L^{T}$ and $L^{F_{T}}$ given in (61) and (62), respectively, together with the Girsanov Theorem. Under $Q^{T}$ they are given by

$$
\begin{aligned}
d F\left(t, T_{1}\right)= & {\left[\sigma_{F}\left(t, T_{1}\right) \sigma_{p}^{*}(t, T)-\int_{E} \delta_{F}\left(t, y, T_{1}\right) \lambda(t, d y)\right] F\left(t, T_{1}\right) d t } \\
& +F\left(t, T_{1}\right) \sigma_{F}\left(t, T_{1}\right) d W_{t}^{T}+F\left(t-, T_{1}\right) \int_{E} \delta_{F}\left(t, y, T_{1}\right) \mu(d t, d y) .
\end{aligned}
$$

The point process $\mu$ has intensity $\lambda^{T}$ under $Q^{T}$, where

$$
\lambda^{T}(t, d y)=\left(1+\delta_{p}(t, y, T)\right) \lambda(t, d y) .
$$

Under $Q^{F_{T}}$ the dynamics of $F\left(t, T_{1}\right)$ are

$$
\begin{aligned}
\frac{d F\left(t, T_{1}\right)}{F\left(t-, T_{1}\right)} & =\left[\sigma_{F}\left(t, T_{1}\right) \sigma_{F}^{*}\left(t, T_{1}\right)+\sigma_{F}\left(t, T_{1}\right) \sigma_{p}^{*}(t, T)-\int_{E} \delta_{F}\left(t, y, T_{1}\right) \lambda(t, d y)\right] d t \\
& +\sigma_{F}\left(t, T_{1}\right) d W_{t}^{F_{T}}+\int_{E} \delta_{F}\left(t, y, T_{1}\right) \mu(d t, d y)
\end{aligned}
$$

Under $Q^{F_{T}}$ the point process $\mu$ has intensity $\lambda^{F_{T}}$, where

$$
\lambda^{F_{T}}(t, d y)=\left(1+\delta_{F}\left(t, y, T_{1}\right)\right)\left(1+\delta_{p}(t, y, T)\right) \lambda(t, d y) .
$$

\subsubsection{The Hilliard-Reis three-factor model.}

Consider again the Hilliard-Reis model defined in (49). The bond price volatilities for this model are given by (see [12])

$$
\sigma_{p}(t, T)=\frac{\sigma_{r}}{\kappa_{r}}\left(e^{-\kappa_{r}(T-t)}-1\right) .
$$

Using the explicit expression for the futures prices, the futures price dynamics can be shown to be (see [11])

$$
d F(t, T)=-F(t, T) \bar{\delta} \lambda d t+F(t, T) \sigma_{F}(t, T) d W_{t}+F(t-, T) \int_{R} y \mu(d t, d y) .
$$

Here, as before, the marked point process $\mu$ has mark space $(R, \mathcal{B})$, where $\mathcal{B}$ is the Borel alebra, and a compensator

$$
\nu(d t, d y)=\lambda d t \frac{1}{\sqrt{2 \pi \eta^{2}}} \cdot \frac{1}{y+1} \exp \left\{-\frac{[\ln (y+1)-\xi]^{2}}{2 \eta^{2}}\right\} d y
$$

for $-1<y<\infty$. Recall that this means that the spot price process will jump according to a Poisson process with intensity $\lambda$ and that if $\delta$ denotes the 
relative jump size, then $1+\delta$ has a log-normal distribution: $\ln (1+\delta) \sim N\left(\xi, \eta^{2}\right)$. Furthermore, $\bar{\delta}=E^{Q}[\delta]$ in (67) denotes the expected relative jump size under $Q$, and we have that

$$
\begin{aligned}
\sigma_{F}(t, T) \sigma_{F}^{*}(t, T) & =\sigma_{S}^{2}+\sigma_{c}^{2} B_{c}^{2}(t, T)+\sigma_{r}^{2} B_{r}^{2}(t, T)+2\left\|\sigma_{S}\right\| \cdot\left\|\sigma_{c}\right\| \rho_{S c} B_{c}(t, T) \\
& +2\left\|\sigma_{S}\right\| \cdot\left\|\sigma_{r}\right\| \rho_{S r} B_{r}(t, T)+2\left\|\sigma_{c}\right\| \cdot\left\|\sigma_{r}\right\| \rho_{c r} B_{c}(t, T) B_{r}(t, T), \\
\sigma_{F}^{*}\left(t, T_{1}\right) \sigma_{p}(t, T) & =-\left\|\sigma_{S}\right\| \cdot\left\|\sigma_{r}\right\| \rho_{S r} B_{r}(t, T)-\left\|\sigma_{c}\right\| \cdot\left\|\sigma_{r}\right\| \rho_{c r} B_{r}(t, T) B_{c}\left(t, T_{1}\right) \\
& -\left\|\sigma_{r}\right\|^{2} B_{r}(t, T) B_{r}\left(t, T_{1}\right) .
\end{aligned}
$$

Specializing the formulas for the dynamics of $F\left(t, T_{1}\right)$ under $Q^{T}$ and $Q^{F_{T}}$ to this case, we see that conditional on that there has been $n$ jumps $F\left(T, T_{1}\right)$ can be written as

$$
\begin{aligned}
F\left(T, T_{1}\right)= & F\left(0, T_{1}\right) \prod_{i=1}^{n}\left(1+\delta_{T_{i}}\right) \times \\
\times & \exp \left\{\int_{0}^{T}\left(\sigma_{F}\left(t, T_{1}\right) \sigma_{p}^{*}(t, T)-\bar{\delta} \lambda-\frac{1}{2} \sigma_{F}\left(t, T_{1}\right) \sigma_{F}^{*}\left(t, T_{1}\right)\right) d t\right. \\
& \left.+\int_{0}^{T} \sigma_{F}\left(t, T_{1}\right) d W_{t}^{T}\right\}
\end{aligned}
$$

where $\delta_{T_{i}}, i=1, \ldots, n$ are i.i.d. random variables and $\ln \left(1+\delta_{T_{i}}\right) \in N\left(\xi, \eta^{2}\right)$ under $Q^{T}$. The variables $\delta_{T_{i}}$ are also independent of $W^{T}$. Alternatively we can write $F\left(T, T_{1}\right)$ as

$$
\begin{aligned}
F\left(T, T_{1}\right)= & F\left(0, T_{1}\right) \prod_{i=1}^{n}\left(1+\Delta_{T_{i}}\right) \times \\
\times & \exp \left\{\int_{0}^{T}\left(\sigma_{F}\left(t, T_{1}\right) \sigma_{p}^{*}(t, T)-\bar{\delta} \lambda+\frac{1}{2} \sigma_{F}\left(t, T_{1}\right) \sigma_{F}^{*}\left(t, T_{1}\right)\right) d t\right. \\
& \left.+\int_{0}^{T} \sigma_{F}\left(t, T_{1}\right) d W_{t}^{F_{T}}\right\},
\end{aligned}
$$

where $\Delta_{T_{i}}, i=1, \ldots, n$ are i.i.d. with $\ln \left(1+\Delta_{T_{i}}\right) \in N\left(\xi+\eta^{2}, \eta^{2}\right)$ under $Q^{F_{T}}$. The variables $\Delta_{T_{i}}$ are also independent of $W^{F_{T}}$. Given this we can express the two probabilities in (66) as follows

$$
\begin{aligned}
Q^{F_{T}}\left(F\left(T, T_{1}\right) \geq K\right) & =\sum_{n=0}^{\infty}\left[\frac{e^{-\lambda^{\prime} T}\left(\lambda^{\prime} T\right)^{n}}{n !}\right] N\left(d_{1 n}\right), \\
Q^{T}\left(F\left(T, T_{1}\right) \geq K\right) & =\sum_{n=0}^{\infty}\left[\frac{e^{-\lambda T}(\lambda T)^{n}}{n !}\right] N\left(d_{2 n}\right),
\end{aligned}
$$


where

$$
\begin{aligned}
d_{1 n} & =\frac{1}{\sqrt{\zeta^{2}+n \eta^{2}}}\left(\ln \left(\frac{F\left(0, T_{1}\right) Y\left(0, T, T_{1}\right)}{K}\right)+n \xi-\bar{\delta} \lambda T+\frac{1}{2} \zeta^{2}+n \eta^{2}\right), \\
d_{2 n} & =d_{1}-\sqrt{\zeta^{2}+n \eta^{2}}, \\
Y\left(t, T, T_{1}\right) & =e^{\int_{t}^{T} \sigma_{F}\left(s, T_{1}\right) \sigma_{p}^{*}(s, T) d s}, \\
\zeta^{2} & =\int_{0}^{T} \sigma_{F}\left(t, T_{1}\right) \sigma_{F}\left(t, T_{1}\right)^{*} d t, \\
\lambda^{\prime} & =\lambda e^{\xi+\eta^{2} / 2} .
\end{aligned}
$$

The price, at date zero, of a European call option with exercise date $T$ and exercise price $K$ written on the futures price with delivery date $T_{1}$ is therefore given by the following formula after some rewriting where we use the fact that $\exp \left\{\xi+\eta^{2} / 2\right\}=\bar{\delta}+1$

$$
\begin{aligned}
C_{F}= & p(0, T) \sum_{n=0}^{\infty}\left[\frac{e^{-\lambda T}(\lambda T)^{n}}{n !}\right]\left[F\left(0, T_{1}\right) Y\left(0, T, T_{1}\right) e^{-\lambda \bar{\delta} T+n \ln (1+\bar{\delta})} N\left[d_{1 n}\right]\right. \\
& \left.-K N\left[d_{2 n}\right]\right] .
\end{aligned}
$$

Here $N$ denotes the cumulative distribution function of a normally distributed random variable with expectation zero and variance one, and $d_{1 n}, d_{2 n}$ and $Y\left(t, T, T_{1}\right)$ have been defined above. This reproduces the results in [11].

\subsubsection{The Gaussian case}

Consider the following model, which is a special case of the model of the previous section, under the martingale measure $Q$.

$$
\begin{aligned}
d f(t, T) & =\alpha_{f}(t, T) d t+\sigma_{f}(t, T) d W_{t}, \\
d F(t, T) & =F(t, T) \alpha_{F}(t, T) d t+F(t, T) \sigma_{F}(t, T) d W_{t} .
\end{aligned}
$$

Here $\sigma_{f}$ and $\sigma_{F}$ are assumed to be deterministic functions of the time parameters, whereas $\alpha_{f}$ and $\alpha_{F}$ are given by the drift conditions (17) and (15), respectively.

Specializing the formulas for the dynamics of $F\left(t, T_{1}\right)$ to this case, we see that $F\left(t, T_{1}\right)$ follows a geometric Brownian motion both under $Q^{T}$ and under $Q^{F_{T}}$. The probabilities can therefore be computed and we find that the price, at date zero, of a European call option with exercise date $T$ and exercise price $K$ written on the futures price with delivery date $T_{1}$ is given by

$$
\begin{aligned}
C_{F}= & p(0, T) F\left(0, T_{1}\right) \exp \left\{\int_{0}^{T} \kappa_{u} d u\right\} N\left[d_{1}\right] \\
& -K p(0, T) N\left[d_{2}\right],
\end{aligned}
$$


where $N$ denotes the cumulative distribution function of a normally distributed random variable with expectation zero and variance one. Furthermore, $d_{1}$ and $d_{2}$ are given by

$$
\begin{aligned}
& d_{1}=\frac{1}{\sqrt{\int_{0}^{T}\left\|\sigma_{F}\left(u, T_{1}\right)\right\|^{2} d u}}\left(\ln \left(\frac{F\left(0, T_{1}\right)}{K}\right)+\int_{0}^{T}\left\{\kappa_{s} d s+\frac{1}{2}\left\|\sigma_{F}\left(s, T_{1}\right)\right\|^{2}\right\} d s\right), \\
& d_{2}=d_{1}-\sqrt{\int_{0}^{T}\left\|\sigma_{F}\left(u, T_{1}\right)\right\|^{2} d u .}
\end{aligned}
$$

The process $\kappa$ was defined in (65). This reproduces the result in [17] (in the referenced work the futures convenience yield and the spot price dynamics are modeled instead of the futures price dynamics, but as was pointed out in Remark 5.1 , deterministic volatilities for the convenience yield and the spot price imply deterministic futures price volatilities, and thus the above pricing formula is applicable).

\subsubsection{Quadratic interest rates}

Consider a model specified by the following equations

$$
\begin{aligned}
d Z_{1}(t) & =\left[\alpha_{1}-\beta_{1} Z_{1}(t)\right] d t+\rho_{1} d W_{1}(t), \\
d Z_{2}(t) & =\left[\alpha_{2}-\beta_{2} Z_{2}(t)\right] d t+\rho_{2} d W_{2}(t), \\
d F(t, T) & =\sigma_{1} F(t, T) d W_{1}(t)+\sigma_{2} F(t, T) d W_{2}(t),
\end{aligned}
$$

and

$$
r(t)=\frac{1}{2}\left(Z_{1}^{2}(t)+Z_{2}^{2}(t)\right) .
$$

The specifications are all made under a martingale measure $Q$, and $W_{1}$ and $W_{2}$ are two independent standard Wiener processes. We assume that $\alpha_{i}, \beta_{i}, \rho_{i}$ and $\sigma_{i}, i=1,2$ are all constants.

For this model the $T$-maturity zero-coupon bond price can be shown to be of the form

$$
p(t, T)=\exp \left\{-\sum_{i=1}^{2}\left[\frac{1}{2} B_{i}(t, T) Z_{i}^{2}(t)+b_{i}(t, T) Z_{i}(t)\right]-c(t, T)\right\},
$$

where $B_{i}, b_{i}, i=1,2$ and $c$ solve the following ordinary differential equations

$$
\begin{aligned}
\frac{\partial B_{i}}{\partial t}=2 \beta_{i} B_{i}+\rho_{i}^{2} B_{i}^{2}-1, & B_{i}(T, T)=0 \\
\frac{\partial b_{i}}{\partial t}-\left(\beta_{i}+\rho_{i}^{2} B_{i}\right) b_{i}+\alpha_{i} B_{i}=0, & b_{i}(T, T)=0,
\end{aligned}
$$




$$
\frac{\partial c}{\partial t}+\sum_{i=1}^{2}\left(\alpha_{i} b_{i}+\frac{1}{2} \rho_{i}^{2}\left[B_{i}-b_{i}^{2}\right]\right)=0, \quad c(T, T)=0
$$

(see [14] for details).

We will now attempt to compute the price, at date zero, of a European call option with exercise date $T$ and exercise price $K$ written on the futures price with delivery date $T_{1}$, using the first pricing formula (63). The dynamics of $Z_{1}$, $Z_{2}$ and $F\left(t, T_{1}\right)$ under $Q^{F}$ are easily found using the dynamics of $L^{F}$ given in (60) together with the Girsanov Theorem. They are

$$
\begin{aligned}
d Z_{1}(t) & =\left[\alpha_{1}+\rho_{1} \sigma_{1}-\beta_{1} Z_{1}(t)\right] d t+\rho_{1} d W_{1}^{F}(t), \\
d Z_{2}(t) & =\left[\alpha_{2}+\rho_{2} \sigma_{2}-\beta_{2} Z_{2}(t)\right] d t+\rho_{2} d W_{2}^{F}(t), \\
d F(t, T) & =\left(\sigma_{1}^{2}+\sigma_{2}^{2}\right) F\left(t, T_{1}\right) d t+\sigma_{1} F(t, T) d W_{1}^{F}(t)+\sigma_{2} F(t, T) d W_{2}^{F}(t),
\end{aligned}
$$

where $W_{1}^{F}$ and $W_{2}^{F}$ are two independent $Q^{F}$-Wiener processes. From this we see that the state variables $Z_{1}$ and $Z_{2}$ are still Gaussian. Using $Q^{F}$ as a martingale measure we can therefore compute bond prices in exactly the same way as before, except for that $\alpha_{i}$ is replaced by $\tilde{\alpha}_{i}=\alpha_{i}+\sigma_{i} \rho_{i}, i=1,2$. These modified bond prices, which we will denote by $\tilde{p}(t, T)$, give us the first expectation in (63). From the above dynamics we see that the futures price $F\left(T, T_{1}\right)$ is log-normally distributed, and hence the second probability in (63) is given by

$$
Q^{T}\left(F\left(T, T_{1}\right) \geq K\right)=N\left[\frac{1}{\left\|\sigma_{F}\right\| \sqrt{T}}\left[\ln \left(\frac{F\left(0, T_{1}\right)}{K}\right)+\frac{1}{2}\left\|\sigma_{F}\right\|^{2} T\right]\right],
$$

where $\sigma_{F}=\left(\sigma_{1}, \sigma_{2}\right)$ and $N$ denotes the cumulative distribution function of a normally distributed random variable with mean zero and variance one.

The Radon-Nikodym derivative of $Q^{T_{F}}$ with respect to $Q^{F}$ can now be written as

$$
R^{T_{F}}=\frac{\exp \left\{-\int_{0}^{T} r_{s} d s\right\}}{\tilde{p}(0, T)} .
$$

This means that the likelihood process is given by

$$
L_{t}^{T_{F}}=\frac{\tilde{p}(t, T)}{B(t) \tilde{p}(0, T)}
$$

(thus, $Q^{T_{F}}$ is the $T$-forward measure "as seen from $Q^{F}$ "). Let $\tilde{B}_{i}, \tilde{b}_{i}, i=1,2$ and $\tilde{c}$ denote the functions you obtain solving the equations (68), (69) and (70), respectively, with $\alpha_{i}$ replaced by $\tilde{\alpha}_{i}=\alpha_{i}+\sigma_{i} \rho_{i}, i=1,2$. Then we have that

$$
\tilde{p}(t, T)=\exp \left\{-\sum_{i=1}^{2}\left[\frac{1}{2} \tilde{B}_{i}(t, T) Z_{i}^{2}(t)+\tilde{b}_{i}(t, T) Z_{i}(t)\right]-\tilde{c}(t, T)\right\} .
$$


Using Itô's formula we then see that the dynamics of $L^{T_{F}}$ are

$$
\begin{aligned}
d L_{t}^{T}= & -L_{t}^{T}\left(\tilde{B}_{1}(t, T) Z_{1}(t)+\tilde{b}_{1}(t, T)\right) \rho_{1} d W_{1}^{F}(t) \\
& -L_{t}^{T}\left(\tilde{B}_{2}(t, T) Z_{2}(t)+\tilde{b}_{2}(t, T)\right) \rho_{2} d W_{2}^{F}(t) .
\end{aligned}
$$

The Girsanov Theorem then gives us the following dynamics under $Q^{T_{F}}$.

$$
\begin{aligned}
d Z_{1}(t)=[ & {\left[\alpha_{1}+\rho_{1} \sigma_{1}-\rho_{1}^{2} \tilde{b}_{1}(t, T)-\left(\beta_{1}+\rho_{1}^{2} \tilde{B}_{1}(t, T)\right) Z_{1}(t)\right] d t } \\
& +\rho_{1} d W_{1}^{T_{F}}(t), \\
d Z_{2}(t)= & {\left[\alpha_{2}+\rho_{2} \sigma_{2}-\rho_{2}^{2} \tilde{b}_{2}(t, T)-\left(\beta_{2}+\rho_{2}^{2} \tilde{B}_{2}(t, T)\right) Z_{2}(t)\right] d t } \\
& +\rho_{2} d W_{2}^{T_{F}}(t), \\
d F(t, T)=[ & \sigma_{1}^{2}+\sigma_{2}^{2}-\left(\tilde{B}_{1}(t, T) Z_{1}(t)+\tilde{b}_{1}(t, T)\right) \rho_{1} \sigma_{1} \\
& \left.-\left(\tilde{B}_{2}(t, T) Z_{2}(t)+\tilde{b}_{2}(t, T)\right) \rho_{2} \sigma_{2}\right] F(t, T) d t \\
& +\sigma_{1} F(t, T) d W_{1}^{T_{F}}(t)+\sigma_{2} F(t, T) d W_{2}^{T_{F}}(t),
\end{aligned}
$$

where $W_{1}^{T_{F}}$ and $W_{2}^{T_{F}}$ are two independent $Q^{T_{F}}$-Wiener processes. Now let $Y(t)=\left(Z_{1}(t), Z_{2}(t), \ln F\left(t, T_{1}\right)\right)^{*}$. If we apply Itô's formula to the third component of this process we see that the process satisfies the following stochastic differential equation

$$
d Y_{t}=\left[\alpha_{Y}(t)-\beta_{Y}(t) Y_{t}\right] d t+\sigma_{Y}(t) d W_{t},
$$

where

$$
\begin{aligned}
& \alpha_{Y}(t)=\left[\begin{array}{c}
\alpha_{1}+\rho_{1} \sigma_{1}-\rho_{1}^{2} \tilde{b}_{1}(t, T) \\
\alpha_{2}+\rho_{2} \sigma_{2}-\rho_{2}^{2} \tilde{b}_{2}(t, T) \\
\frac{1}{2}\left\|\sigma_{F}\right\|^{2}-\rho_{1} \sigma_{1} \tilde{b}_{1}(t, T)-\rho_{2} \sigma_{2} \tilde{b}_{2}(t, T)
\end{array}\right], \\
& \beta_{Y}(t)=\left[\begin{array}{ccc}
\beta_{1}+\rho_{1}^{2} \tilde{B}_{1}(t, T) & 0 & 0 \\
0 & \beta_{2}+\rho_{2}^{2} \tilde{B}_{2}(t, T) & 0 \\
\rho_{1} \sigma_{1} \tilde{B}_{1}(t, T) & \rho_{2} \sigma_{2} \tilde{B}_{2}(t, T) & 0
\end{array}\right],
\end{aligned}
$$

and

$$
\sigma_{Y}(t)=\left[\begin{array}{cc}
\rho_{1} & 0 \\
0 & \rho_{2} \\
\sigma_{1} & \sigma_{2}
\end{array}\right]
$$

Suppose that $Y_{0}$ is deterministic. We then see that $Y$ has a three dimensional normal distribution, where the mean $m(t)=E^{T_{F}}\left[Y_{t}\right]$ and the covariance matrix $V=E\left[\left\|\left(Y_{t}-m(t)\right)\right\|^{2}\right]$ are obtained as the solutions to the following linear 
equations (see for instance [15]).

$$
\begin{aligned}
& \dot{m}(t)=-\beta_{Y}(t) m(t)+\alpha_{Y}(t), \\
& \dot{V}(t)=-\beta_{Y}(t) V(t)-V(t) \beta_{Y}^{*}(t)+\sigma_{Y}(t) \sigma_{Y}^{*}(t) .
\end{aligned}
$$

The probability in the pricing formula (63) is therefore given by

$$
Q^{T_{F}}\left(F\left(T, T_{1}\right) \geq K\right)=N\left[\frac{1}{\sqrt{V_{33}(T)}}\left[\ln \left(\frac{F\left(0, T_{1}\right)}{K}\right)+m_{3}(T)\right]\right] .
$$

\section{Inverting the term structure}

Consider a given finite dimensional factor model for the futures term structure, say of the form

$$
\begin{aligned}
F(t, T) & =H_{F}\left(t, Z_{t}, T\right) \\
d Z_{t} & =\alpha_{Z}\left(t, Z_{t}\right) d t+\sigma_{Z}\left(t, Z_{t}\right) d W_{t}+\int_{E} \delta_{Z}\left(t, Z_{t-}, y\right) \mu(d t, d y),
\end{aligned}
$$

under the martingale measure $Q$. For a given initial value $z_{0}$ of $Z_{0}$ the model will produce the theoretical initial term structure $F(0, T)=H_{F}\left(0, z_{0}, T\right)$. Assuming that we have a liquid futures market for all delivery dates, the market will provide us with an observed initial futures term structure $F^{\dagger}(0, T)$, and we would of course like our theoretical initial term structure to coincide with the observed one. We would thus like to choose the parameters in the $Z$-dynamics such that

$$
F(0, T)=F^{\dagger}(0, T), \quad \forall T \geq 0,
$$

and since this is an infinite dimensional system of equations, we will need an infinite dimensional parameter vector. The entire project is thus completely parallel to that of inverting the yield curve in interest rate theory.

\subsection{Conditionally affine models}

The general problem of when and how it is possible to fit an arbitrarily given initial term structure, for a parameterized family of futures price models, is a very hard one and there seems to be no strong general results (see however [16]). Here we will instead present a nontrivial particular class of models for which the initial term structure in fact can be inverted, and it turns out that most existing factor models belong to this class. The class is characterized by the facts that the spot price $S$ is one of the factors, and we furthermore impose a particular structure on the factor dynamics. See also [3], where basically the same approach was applied to interest rate theory.

Definition 6.1 We say that a factor model for futures prices is a conditionally affine model if the following conditions hold. 
- The factor vector can be decomposed as $\left(S_{t}, Z_{t}\right)$ where $Z \in R^{d}$.

- The factor dynamics are of the form

$$
\begin{aligned}
d S_{t} & =S_{t}\left\{f\left(Z_{t}\right)-\kappa \ln S_{t}\right\} d t+S_{t} g\left(Z_{t}\right) d W_{t} \\
& +S_{t-} \int_{E} \delta_{S}\left(t, Z_{t-}, y\right) \mu(d t, d y) \\
d Z_{t} & =a\left(Z_{t}\right) d t+b\left(Z_{t}\right) d W_{t}+\int_{E} \delta_{z}\left(t, Z_{t-}, y\right) \mu(d t, d y) .
\end{aligned}
$$

Here $\mu$ is a point process, $W$ is an m-dimensional Wiener process and $\kappa$ is a (typically positive) real number. The functions $a, b, f$ and $g$ are nonlinear functions of appropriate dimensions ( $Z$ is viewed as a column vector process). The jump volatility functions $\delta_{S}(t, z, y)$ and $\delta_{z}(t, z, y)$ are assumed to be deterministic functions of the variables $t, z$ and $y$ with $\delta_{S}>-1$.

Given a conditionally affine model $(S, Z)$ as above, as well as a deterministic $R$-valued function $\varphi(t)$, the corresponding perturbed model $\left(S^{\varphi}, Z\right)$ is defined by the relations

$$
\begin{aligned}
d S_{t}^{\varphi} & =S_{t}\left\{\varphi(t)+f\left(Z_{t}\right)-\kappa \ln S_{t}^{\varphi}\right\} d t+S_{t} g\left(Z_{t}\right) d W_{t} \\
& +S_{t-} \int_{E} \delta_{S}\left(t, Z_{t-}, y\right) \mu(d t, d y) \\
d Z_{t} & =a\left(Z_{t}\right) d t+b\left(Z_{t}\right) d W_{t}+\int_{E} \delta_{z}\left(t, Z_{t-}, y\right) \mu(d t, d y) .
\end{aligned}
$$

The perturbed model is assumed to have the same initial data as the original model. The futures prices generated by the original model and the perturbed model are denoted by $F(t, T)$ and $F^{\varphi}(t, T)$ respectively.

Note that the dynamics of the $Z$ process does not involve $S$ at all. The deeper significance of the $S$-dynamics is that with this particular form, and for a given $Z$-trajectory, we can write $S_{t}=e^{\xi_{t}}$ where $\xi$ satisfies a linear SDE. The above dynamics are thus (conditional on the $Z$-trajectory) the natural extension of the standard Black-Scholes stock price dynamics (including jumps). We also note that $Z$ is not affected by the choice of $\varphi$ and that the original model corresponds to $\varphi=0$.

Given an initial (observed) term structure, $\left\{F^{\dagger}(0, T) ; T \geq 0\right\}$ the problem is to see if it is possible to choose $\varphi$ in such a way that $F^{\varphi}(0, T)=F^{\dagger}(0, T)$ for all $T \geq 0$. The reader will note the similarity between this perturbation approach and the way in which Hull-White extend the Vasicek short rate model in order to invert the yield curve. We have the following strong result. 
Proposition 6.1 Let $F^{\dagger}(0, T)$ be any smooth (i.e. $\left.C^{1}\right)$ initial term structure such that $F^{\dagger}(0,0)=F^{0}(0,0)$. Define the function $H$ by

$$
H(T)=\ln \left(\frac{F^{\dagger}(0, T)}{F^{0}(0, T)}\right)
$$

Then the following hold:

- The perturbed model can be fitted to $F^{\dagger}$. In fact, by choosing $\varphi$ as

$$
\varphi(t)=H^{\prime}(t)+\kappa H(t)
$$

we have

$$
F^{\varphi}(0, T)=F^{\dagger}(0, T), \quad \forall T \geq 0 .
$$

- With $S_{0}^{0}=S_{0}^{\varphi}$ and with the above choice of $\varphi$ we have, for all $0 \leq t \leq T$,

$$
F^{\varphi}(t, T)=\frac{F^{\dagger}(0, T)}{F^{0}(0, T)} F^{0}(t, T) .
$$

- Assume that the short rate $r$ is deterministic. For any contingent Tclaim of the form $\Phi\left(S_{T}, Z_{T}\right)$ we denote the corresponding arbitrage free pricing functions by $P^{0}(t, s, z)$ and $P^{\varphi}(t, s, z)$ for the original model and the perturbed model respectively. Then the following relation hold.

$$
P^{\varphi}(t, s, z)=P^{0}\left(t, s e^{\int_{t}^{T} e^{-\kappa(T-u)} \varphi(u) d u}, z\right) .
$$

- Assume in particular that that $\varphi$ is chosen such that the initial term structure is completely fitted as above. Then we have

$$
P^{\varphi}(t, s, z)=P^{0}\left(t, s \cdot\left\{H(T)-e^{-\kappa(T-t)} H(t)\right\}, z\right) .
$$

Remark 6.1 The point of (76)-(77) is that if we have computed derivatives pricing formulas in the original model, then these formulas can be used in the perturbed model, by simply modifying the value of the observed spot price.

Proof. Defining $\xi$ by $\xi=\ln S$ it is easy to see that

$$
\begin{aligned}
d \xi_{t}^{\varphi} & =\left\{-\kappa \xi_{t}^{\varphi}+\varphi(t)+f_{\xi}\left(Z_{t}\right)\right\} d t+g\left(Z_{t}\right) d W_{t} \\
& +\int_{E} \delta_{\xi}\left(t, Z_{t-}, y\right) \mu(d t, d y),
\end{aligned}
$$

where

$$
\begin{aligned}
f_{\xi}(z) & =f(z)-\frac{1}{2} g^{2}(z), \\
\delta_{\xi}(t, z, y) & =\ln \left\{1+\delta_{S}(t, z, y)\right\} .
\end{aligned}
$$


Using the Itô formula one verifies readily that

$$
\begin{aligned}
\xi_{T}^{\varphi}= & e^{-\kappa T} \xi_{0}^{\varphi}+\int_{0}^{T} e^{-\kappa(T-u)} \varphi(u) d u+\int_{0}^{T} e^{-\kappa(T-u)} f_{\xi}\left(Z_{u}\right) d u \\
& +\int_{0}^{T} e^{-\kappa(T-u)} g\left(Z_{u}\right) d W_{u} \\
& +\int_{0}^{T} \int_{E} e^{-\kappa(T-u)} \delta_{\xi}\left(u, Z_{u-}, y\right) \mu(d u, d y)
\end{aligned}
$$

and, since $\xi_{0}^{\varphi}=\xi_{0}^{0}=\ln S_{0}$, we have

$$
\xi_{T}^{\varphi}=\xi_{T}^{0}+\int_{0}^{T} e^{-\kappa(T-u)} \varphi(u) d u,
$$

and hence,

$$
S_{T}^{\varphi}=S_{T}^{0} \cdot e^{\int_{0}^{T} e^{-\kappa(T-u)} \varphi(u) d u} .
$$

From this we obtain, for all $t$ and $T$ with $0 \leq t \leq T$

$$
\begin{aligned}
F^{\varphi}(t, T) & =E^{Q}\left[S_{T}^{\varphi} \mid \mathcal{F}_{t}\right]=e^{\int_{0}^{T} e^{-\kappa(T-u)} \varphi(u) d u} E^{Q}\left[S_{T}^{0} \mid \mathcal{F}_{t}\right] \\
& =e^{\int_{0}^{T} e^{-\kappa(T-u)} \varphi(u) d u} F^{0}(t, T) .
\end{aligned}
$$

Thus, in order to fit the given initial term structure $F^{\dagger}$ we have to find $\varphi$ such that

$$
F^{\dagger}(0, T)=e^{\int_{0}^{T} e^{-\kappa(T-u)} \varphi(u) d u} F^{0}(0, T) .
$$

This equation can, with $H$ defined as above, be written as

$$
\int_{0}^{T} e^{\kappa u} \varphi(u) d u=e^{\kappa T} H(T)
$$

and, taking the $T$-derivative, we obtain (74). The relation (75) follows immediately from (79)-(80).

If $\Phi\left(S_{T}, Z_{T}\right)$ is a contingent $T$-claim, then the corresponding arbitrage free price process $\Pi^{\varphi}(t ; \Phi)$ for the perturbed model is given by

$$
\Pi^{\varphi}(t ; \Phi)=e^{-\int_{t}^{T} r_{u} d u} E^{Q}\left[\Phi\left(S_{T}^{\varphi}, Z_{T}\right) \mid \mathcal{F}_{t}\right] .
$$

From the Markovian setup we have in fact

$$
E^{Q}\left[\Phi\left(S_{T}^{\varphi}, Z_{T}\right) \mid \mathcal{F}_{t}\right]=P_{0}^{\varphi}\left(t, S_{t}^{\varphi}, Z_{t}\right),
$$

where the real valued function $P_{0}^{\varphi}: R_{+} \times R_{+} \times R^{d} \rightarrow R$ is defined by

$$
P_{0}^{\varphi}(t, s, z)=E^{Q}\left[\Phi\left(S_{T}^{\varphi}, Z_{T}\right) \mid S_{t}^{\varphi}=s, Z_{t}=z\right] .
$$


The pricing function $P^{\varphi}$ is thus given by $P^{\varphi}(t, s, z)=\exp \left\{\int_{t}^{T} r(u) d u\right\} P_{0}^{\varphi}(t, s, z)$. Now, given that $S_{t}^{\varphi}=s$, we obtain as above,

$$
\begin{aligned}
S_{T}^{\varphi}= & s \cdot e^{\int_{t}^{T} e^{a(T-u)} \varphi(u) d u} \cdot \exp \left\{e^{-\kappa(T-t)}+\int_{t}^{T} e^{-\kappa(T-u)}\left\{\varphi(u)+f_{\xi}\left(Z_{u}\right)\right\} d u\right\} \\
& \times \exp \left\{\int_{0}^{T} e^{-\kappa(T-u)} g\left(Z_{u}\right) d W_{u}\right\} \\
& \times \exp \left\{\int_{0}^{T} \int_{E} e^{-\kappa(T-u)} \delta_{\xi}\left(u, Z_{u-}, y\right) \mu(d u, d y)\right\}
\end{aligned}
$$

From direct inspection of this formula we see that the distribution of $\left(S_{T}^{\varphi}, Z_{T}\right)$ given $S_{t}^{\varphi}=s$ and $Z_{t}=z$ is identical with the distribution of $\left(S_{T}^{0}, Z_{T}\right)$ given $S_{t}^{\varphi}=s \cdot e^{\int_{t}^{T} e^{-\kappa(T-u)} \varphi(u) d u}$ and $Z_{t}=z$. This proves $(76)-(77)$.

\subsection{An example}

To exemplify the theory above we now give a brief sketch of how to fit the Schwartz Three Factor Model in Section 4.2.4 to an initial future price curve $F^{\dagger}(0, T)$. The perturbed model is given by

$$
\begin{aligned}
d S_{t} & =\left(r_{t}+\varphi(t)-c_{t}\right) S_{t} d t+S_{t} \sigma_{S} d W_{t} \\
d c_{t} & =\kappa_{c}\left(\alpha_{c}-c_{t}\right) d t+\sigma_{c} d W_{t} \\
d r_{t} & =\kappa_{r}\left(\alpha_{r}-r_{t}\right) d t+\sigma_{r} d W_{t}
\end{aligned}
$$

and $\varphi$ is obtained from Proposition 6.1 as

$$
\varphi(t)=\frac{\partial}{\partial t} \ln F^{\dagger}(0, t)+f(0, t)-\frac{\partial}{\partial t} B_{c}(0, t) c_{0}-\frac{\partial}{\partial t} \bar{A}(0, t)
$$

Here $f(0, t)$ denotes the forward rates in the Vasiček short rate model, whereas $B_{c}$ and $\bar{A}$ are given in Remark 4.2.

It is worth noticing that with this perturbation, the process $c_{t}$ no longer has the interpretation of being the spot convenience yield. The spot convenience yield $c^{\varphi}$ in the perturbed model is instead given by $c_{t}^{\varphi}=c_{t}-\varphi(t)$. Using $S, c^{\varphi}$ and $r$ as state variables we easily obtain the alternative dynamics

$$
\begin{aligned}
d S_{t} & =\left(r_{t}-c_{t}^{\varphi}\right) S_{t} d t+S_{t} \sigma_{S} d W_{t}, \\
d c_{t}^{\varphi} & =\kappa_{c}\left[\alpha_{c}-\varphi(t)-\kappa_{c}^{-1} \varphi^{\prime}(t)-c_{t}^{\varphi}\right] d t+\sigma_{c} d W_{t}, \\
d r_{t} & =\kappa_{r}\left(\alpha_{r}-r_{t}\right) d t+\sigma_{r} d W_{t} .
\end{aligned}
$$




\section{References}

[1] Amin, K. I., Ng, V., And Pirrong, S. Valuing energy derivatives. In Managing Energy Price Risk. Risk Publications, 1995, pp. 57-70.

[2] BJörk, T. Arbitrage Theory in Continuous Time. Oxford University Press, 1998.

[3] BJÖrk, T., AND Hyll, M. On the inversion of the yield curve. Working paper, Stockholm School of Economics, 2000.

[4] Björk, T., Kabanov, Y., And Runggaldier, W. Bond market structure in the presence of a marked point process. Mathematical Finance 7, 2 (1995), 211-239.

[5] Cortazar, G., And Schwartz, E. The valuation of commodity contingent claims. Journal of Derivatives (1994), 27-39.

[6] Cox, J., Ingersold, J., And Ross, S. A theory of the term structure of interest rates. Econometrica 53 (1985), 385-408.

[7] Duffie, D., And Kan, R. A yield factor model of interest rates. Mathematical Finance 6, 4 (1996), 379-406.

[8] Duffie, D., Pan, J., And Singleton, K. Transform analysis and asset pricing for affine jump diffusions. Forthcoming in Econometrica (2000).

[9] Geman, H., El Karoui, N., and Rochet, J.-C. Changes of numéraire, changes of probability measure and option pricing. Journal of Applied Probability 32 (1995), 443-458.

[10] Gibson, R., And Schwartz, E. Stochastic convenience yield and the pricing of oil contingent claims. Journal of Finance 45, 3 (1990), 959-976.

[11] Hilliard, J., AND Reis, J. Valuation of commodity futures and options under stochastic convenience yields, interest rates, and jump diffusion in the spot. JFQA 33, 1 (1998), 61-86.

[12] Hull, J., And White, A. Pricing interest-rate-derivative securities. Review of Financial Studies 3 (1990), 573-592.

[13] Jacod, J., And Shiryaev, A. Limit Theorems for Stochastic Processes. Springer verlag, 1987.

[14] Jamshidian, F. Bond, futures and option evaluation in the quadratic interest rate model. Applied Mathematical Finance 3 (1996), 93-115.

[15] Karatzas, I., And Shreve, S. Brownian motion and stochastic calculus. Springer, 1991.

[16] LAndÉn, C. Spot price realizations of futures price term structures. Tech. rep., Department of Mathematics, KTH, Stockholm, 2000. 
[17] Miltersen, K., And Schwartz, E. Pricing of options on commodity futures with stochastic term structures of convenience yields and interest rates. JFQA 33 (1998), 33-59.

[18] Schwartz, E. The stochastic behaviour of commodity prices. Journal of Finance 52, 3 (1997), 923-973.

[19] VAsičEK, O. An equilibrium characterization of the term structure. Journal of Financial Economics 5, 3 (1977), 177-188. 